\title{
How Quantum Evolution with Memory is Generated in a Time-Local Way
}

\author{
K. Nestmann $\oplus^{1,2}$ V. Bruch ${ }^{1,2}$ and M. R. Wegewijs $\oplus^{1,2,3}$ \\ ${ }^{1}$ Institute for Theory of Statistical Physics, RWTH Aachen, 52056 Aachen, Germany \\ ${ }^{2}$ JARA-FIT, 52056 Aachen, Germany \\ ${ }^{3}$ Peter Grünberg Institut, Forschungszentrum Jülich, 52425 Jülich, Germany
}

(Received 11 February 2020; revised 17 December 2020; accepted 23 March 2021; published 24 May 2021)

\begin{abstract}
Two widely used but distinct approaches to the dynamics of open quantum systems are the Nakajima-Zwanzig and time-convolutionless quantum master equation, respectively. Although both describe identical quantum evolutions with strong memory effects, the first uses a time-nonlocal memory kernel $\mathcal{K}$, whereas the second achieves the same using a time-local generator $\mathcal{G}$. Here we show that the two are connected by a simple yet general fixed-point relation: $\mathcal{G}=\hat{\mathcal{K}}[\mathcal{G}]$. This allows one to extract nontrivial relations between the two completely different ways of computing the time evolution and combine their strengths. We first discuss the stationary generator, which enables a Markov approximation that is both nonperturbative and completely positive for a large class of evolutions. We show that this generator is not equal to the low-frequency limit of the memory kernel, but additionally "samples" it at nonzero characteristic frequencies. This clarifies the subtle roles of frequency dependence and semigroup factorization in existing Markov approximation strategies. Second, we prove that the fixed-point equation sums up the time-domain gradient or Moyal expansion for the time-nonlocal quantum master equation, providing nonperturbative insight into the generation of memory effects. Finally, we show that the fixed-point relation enables a direct iterative numerical computation of both the stationary and the transient generator from a given memory kernel. For the transient generator this produces nonsemigroup approximations which are constrained to be both initially and asymptotically accurate at each iteration step.
\end{abstract}

DOI: 10.1103/PhysRevX.11.021041

Subject Areas: Quantum Information,

Statistical Physics

\section{INTRODUCTION}

It is well known that the dynamics $\rho\left(t_{0}\right) \rightarrow \rho(t)$ of the state of an open quantum system initially uncorrelated with its environment can be described equivalently by two exact but fundamentally different quantum master equations (QMEs). On the one hand, the Nakajima-Zwanzig [1,2] time-nonlocal QME,

$$
\frac{d}{d t} \rho(t)=-i \int_{t_{0}}^{t} d s \mathcal{K}(t, s) \rho(s)
$$

features a memory kernel $\mathcal{K}(t, s)$ with separate dependence on all intermediate times $s \in\left[t_{0}, t\right]$. Here memory is simply understood as retardation. On the other hand, the time-convolutionless time-local QME of Tokuyama and Mori [3,4],

Published by the American Physical Society under the terms of the Creative Commons Attribution 4.0 International license. Further distribution of this work must maintain attribution to the author(s) and the published article's title, journal citation, and DOI.

$$
\frac{d}{d t} \rho(t)=-i \mathcal{G}\left(t, t_{0}\right) \rho(t),
$$

has a generator $\mathcal{G}\left(t, t_{0}\right)$, which incorporates the memory integral into its dependence on the current time $t$ and the initial time $t_{0}$. Both equations are widely used in the areas of quantum transport, chemical kinetics, quantum optics, and quantum-information theory. In the absence of coupling to the environment and external driving, there is a simple relation between the two, $\mathcal{K}(t-s)=L \bar{\delta}(t-s)$ is time local [5] while $\mathcal{G}\left(t-t_{0}\right)=L$ is time independent, such that both reproduce the Liouville-von Neumann equation $(d / d t) \rho(t)=-i[H, \rho(t)]=:-i L \rho(t)$ for a closed system. In some well-understood cases, for example, in the limit of weak coupling [6], high temperature [7,8], and limits of singular coupling [9-11], this simple relation continues to hold, since the Liouvillian $L$ is merely extended by a constant term accounting for dissipative effects, $\mathcal{K}(t-s)=\mathcal{G} \bar{\delta}(t-s)$ with $\mathcal{G}=L+i \mathcal{D}$. In these cases the time-local QME takes the celebrated GoriniKossakowski-Sudarshan-Lindblad (GKSL) [12,13] form. We are interested instead in the generic relation between $\mathcal{G}$ and $\mathcal{K}$ beyond these simple cases, where strong coupling, low temperature, driving and nonequilibrium nontrivially 
compete and both dissipation and memory effects are strong. Not only are these phenomena important for understanding the disturbance of quantum devices in applications, it is also of intrinsic interest to study them in the highly controlled engineered structures available nowadays [14-16].

An immediate question is why one would bother to convert between two equivalent QMEs, if instead one could just solve the equation one has in hand for $\rho(t)$. Careful consideration of this question supports a complementary view [17-19]. Typically $\mathcal{K}$ is easier to compute and advanced methods have been developed to obtain it analytically $[20,21]$ and numerically $[22,23]$ with successful applications to nontrivial models [24-27] covering transient and stationary dynamics, as well as counting statistics [28-30] of observables. The direct computation of $\mathcal{G}$ using the time-convolutionless formalism [6,31-35] is typically more challenging.

However, when solving the time-nonlocal equation (1), taking the frequency dependence of the memory kernel into account (retardation) may in fact lead one to first construct a corresponding time-local equation (2) [28,36-38], which is subsequently solved. Moreover, the generator $\mathcal{G}$ by itself is of particular interest: it allows one to infer important properties of the propagator,

$$
\rho(t)=\Pi\left(t, t_{0}\right) \rho\left(t_{0}\right),
$$

which are very difficult to see otherwise. For example, the complete positivity (CP) of the propagator $\Pi\left(t, t_{0}\right)$, fundamental to its physical legitimacy, may in many situations beyond the GKSL case be inferred [39-41] explicitly from a time-dependent canonical form [42] of $\mathcal{G}$. This is important for constructing both well-defined phenomenological QMEs [17,18] and microscopic models that obey prescribed QMEs [43]. Related to this is that $\mathcal{G}$ often has a clear operational meaning in terms of quantum jumps, which makes it advantageous for stochastic simulations. For the same reason, it is often employed to construct noise models in quantum-information theory, an issue of everincreasing importance. Despite continued efforts, the above is much more complicated to achieve when using $\mathcal{K}$, via either its microscopic coupling expansion [44] or a legitimate-pair decomposition [45,46] encompassing broad classes of models (semi-Markov [47,48], collision models [49], and beyond [50,51]). A further key property that can be inferred directly from $\mathcal{G}$ is its so-called divisibility using its canonical jump rates $[39,41,52]$ and jump operators [53,54]. Again, this seems prohibitively difficult when using $\mathcal{K}$ [55]. Divisibility is not only of key importance for the precise characterization of quantum nonMarkovianity, see Sec. VI, a concept much broader [56] than memory understood as retardation. It also features in quantum coding [57,58] and tomography [59], key distribution [60], teleportation [61], and work extraction by erasure [62]; see Sec. VI. Finally, the time-local nature of Eq. (2) featuring $\mathcal{G}$ is crucial to access geometric [63-65] and possible topological $[66,67]$ phases in open-system evolution with applications to pumping, full-counting statistics [68], fluctuation relations [69], entropy production [70,71], and quantum thermodynamics [72-74]. Thus, although in principle Eqs. (1) and (2) are obviously equivalent, there are many reasons for explicitly understanding their general relation, motivating recent work [75].

The relation between $\mathcal{G}$ and $\mathcal{K}$ has already been investigated for time-translational systems in the stationary limit $t_{0} \rightarrow-\infty$. References $[28,37,38]$ discussed this using a memory expansion, i.e., a gradient or Moyal expansion [76-79] in the time domain applied to the density operator. Such expansions are well developed [80,81] for Wigner and Green functions $[78,79]$ and time-dependent density-functional theory $[82,83]$. The mentioned works indicated that the naive physical intuition that the long-time limit of QME (2) is equivalent to the low-frequency approximation to QME (1) is wrong: The stationary generator $\mathcal{G}(\infty)=$ $\lim _{t \rightarrow \infty} \mathcal{G}(t)$ does not coincide with the zero-frequency limit $\hat{\mathcal{K}}(0)=\lim _{\omega \rightarrow 0} \hat{\mathcal{K}}(\omega)$ of the Laplace-transformed memory kernel:

$$
\hat{\mathcal{K}}(\omega)=\lim _{t_{0} \rightarrow-\infty} \int_{t_{0}}^{t} d t \mathcal{K}\left(t-t_{0}\right) e^{i \omega\left(t-t_{0}\right)} .
$$

As a result, "natural" Markovian semigroup approximations set up within the approach based on Eqs. (1) and (2), using the exact $\hat{\mathcal{K}}(0)$ and $\mathcal{G}(\infty)$, respectively, turn out to be distinct. This difference has proven to be important in perturbative studies beyond weak coupling $[28,37,38]$, and is even crucial for measurement backaction $[84,85]$. From these studies the difference between $\hat{\mathcal{K}}(0)$ and $\mathcal{G}(\infty)$ appears to be very complicated. This also ties in with the broader [56] discussion of non-Markovianity, where the interesting connection between divisibility, statistical discrimination [54,86,87], and information flow [41,5254,88-90] continues to develop [19,91].

A further important step was provided by the proof in Ref. [35] that $\hat{\mathcal{K}}(0)$ and $\mathcal{G}(\infty)$, despite their difference, both have the exact stationary state as a right zero eigenvector. However, this work was restricted to master equations for probabilities and also left unanswered the relation between the full eigenspectra of $\mathcal{G}(\infty)$ and $\hat{\mathcal{K}}(\omega)$, which is one of the results established in the present paper. Such relations are of interest since these eigenspectra enter advanced calculations $[24,26,27]$ and provide insight into the time evolution [92], just as the eigenspectra of Hamiltonians do for the evolution of closed systems. Similar exact relations among the eigenvectors of the memory kernel $\mathcal{K}$ proved to be very useful for simplifying the complicated calculations for strongly coupled, strongly interacting quantum dots far out of equilibrium $[7,93,94]$. 
Thus, it is a pressing question of both fundamental and practical interest how the time-local generator is related to the time-nonlocal memory kernel for a general finitedimensional open quantum system. The central result of this paper, presented in Sec. II, is that this relation takes the surprisingly simple form of a functional fixed-point equation $\mathcal{G}\left(t, t_{0}\right)=\hat{\mathcal{K}}[\mathcal{G}]\left(t, t_{0}\right)$. Importantly, it applies to transient dynamics and allows for arbitrary driving.

In Sec. III we first explore the implications for timetranslational systems in the long-time limit, where the stationary generator becomes the fixed point of a simpler function of superoperators, $\mathcal{G}(\infty)=\hat{\mathcal{K}}(\mathcal{G}(\infty))$. This leads to the key insight that $\mathcal{G}(\infty)$ "samples" the memory kernel $\hat{\mathcal{K}}(\omega)$ at a finite number of frequencies. This completely defines $\mathcal{G}(\infty)$ and significantly simplifies the connection between the mentioned distinct Markovian approximations. The sampled frequencies are shown to be exact timeevolution poles, well known from the Laplace resolvent technique $[20,21,24]$ for solving the time-nonlocal equation (1), an entirely different procedure. The transformation connecting eigenvectors of $\mathcal{G}(\infty)$ and $\hat{\mathcal{K}}(\omega)$ is found to be related to so-called initial-slip correction procedures [95-99]. We show that both the stationary and the transient fixed-point equation are self-consistent expressions for the solution of the memory expansion discussed in Refs. $[28,37,38]$ by explicitly constructing and summing this series.

In Sec. IV we show that the fixed-point equation can be turned into two separate iterative numerical approaches for obtaining the transient and the stationary generator, respectively, from a given memory kernel. This provides a new starting point for hybrid approaches in which the results of advanced time-nonlocal calculations [21-24] can be plugged into the time-local formalisms directly, bypassing the solution $\Pi\left(t, t_{0}\right)$ that ties Eqs. (1) and (2) together. Reference [23] numerically addressed the converse problem of extracting $\mathcal{K}$ from an evolution generated by $\mathcal{G}$, which analytically seems to be more complicated.

Finally, in Sec. V we explicitly illustrate the derived relation between $\mathcal{K}$ and $\mathcal{G}$ on two nonperturbative examples. For the exactly solvable dissipative Jaynes-Cummings model $[6,17,100,101]$ we show how it deals with nontrivial singularities of $\mathcal{G}\left(t, t_{0}\right)$ in time. The fermionic resonant level model $[94,102]$ with its richer time-dependent algebraic structure further showcases the nontrivial connection between a time-local and -nonlocal description.

We summarize in Sec. VI and outline how our result may enable progress in various directions. Throughout the paper, we set $\hbar=k_{B}=1$.

\section{FUNCTIONAL FIXED-POINT EQUATION}

By definition the generator $\mathcal{G}\left(t, t_{0}\right)$ and the memory kernel $\mathcal{K}(t, s)$ are related by the fact that they produce the same dynamics $\Pi\left(t, t_{0}\right)$ [Eq. (3)]. To derive a direct relation we start from the time-local QME for the propagator,

$$
\frac{d}{d t} \Pi\left(t, t_{0}\right)=-i \mathcal{G}\left(t, t_{0}\right) \Pi\left(t, t_{0}\right),
$$

with initial condition given by identity, $\Pi\left(t_{0}, t_{0}\right)=\mathcal{I}$. The generator can be obtained from the above equation assuming the inverse propagator exists [103-106],

$$
-i \mathcal{G}\left(t, t_{0}\right)=\left[\frac{d}{d t} \Pi\left(t, t_{0}\right)\right] \Pi\left(t, t_{0}\right)^{-1},
$$

postponing discussion of singular time points to Sec. VA. The equivalent time-nonlocal QME,

$$
\frac{d}{d t} \Pi\left(t, t_{0}\right)=-i \int_{t_{0}}^{t} d s \mathcal{K}(t, s) \Pi\left(s, t_{0}\right),
$$

when inserted into Eq. (6), gives

$$
\mathcal{G}\left(t, t_{0}\right)=\int_{t_{0}}^{t} d s \mathcal{K}(t, s) \Pi\left(s, t_{0}\right) \Pi\left(t, t_{0}\right)^{-1} .
$$

The key step to connect these two approaches originating in statistical physics [1-4] is to recognize the expression for the divisor $\Pi\left(t, s \mid t_{0}\right):=\Pi\left(t, t_{0}\right) \Pi\left(s, t_{0}\right)^{-1}$. This quantity is well known from the quantum-information approach to open-system dynamics, which focuses on complete positivity and divisibility properties $[12,13,39,41,52]$. The divisor describes the propagation $\rho(t)=\Pi\left(t, s \mid t_{0}\right) \rho\left(s \mid t_{0}\right)$ starting from a state at an intermediate time $s \in\left[t_{0}, t\right]$ produced by the same evolution, $\rho\left(s \mid t_{0}\right)=\Pi\left(s, t_{0}\right) \rho\left(t_{0}\right)$. This results in the parametric dependence on $t_{0}$. The divisor obeys the same time-local QME, $d \Pi\left(t, s \mid t_{0}\right) / d t=$ $-i \mathcal{G}\left(t, t_{0}\right) \Pi\left(t, s \mid t_{0}\right)$, with initial condition $\Pi\left(s, s \mid t_{0}\right)=\mathcal{I}$ for all $s \in\left[t_{0}, t\right]$. The inverse of its formal solution,

$$
\Pi\left(s, t \mid t_{0}\right)=\left[\Pi\left(t, s \mid t_{0}\right)\right]^{-1}=\mathcal{T}_{\rightarrow} e^{i \int_{s}^{t} d \tau \mathcal{G}\left(\tau, t_{0}\right)},
$$

for $t_{0} \leq s \leq t$, involves anti-time ordering denoted by $\mathcal{T}_{\rightarrow}$. Inserted into Eq. (8), we find the main result of the paper:

$$
\mathcal{G}\left(t, t_{0}\right)=\hat{\mathcal{K}}[\mathcal{G}]\left(t, t_{0}\right) .
$$

The time-local generator is a fixed point of a functional which maps a superoperator function of time $X\left(t, t_{0}\right)$ to another such function:

$$
\hat{\mathcal{K}}[X]\left(t, t_{0}\right):=\int_{t_{0}}^{t} d s \mathcal{K}(t, s) \mathcal{T}_{\rightarrow} e^{i \int_{s}^{t} d \tau X\left(\tau, t_{0}\right)} .
$$

This functional is closely related to the ordinary Laplace transform (4) of the memory kernel $\mathcal{K}(t-s)$, to which it reduces for constant $c$-number functions of time $X=\omega \mathcal{I}$ in the limit $t-t_{0} \rightarrow \infty$ for time-translational systems. 


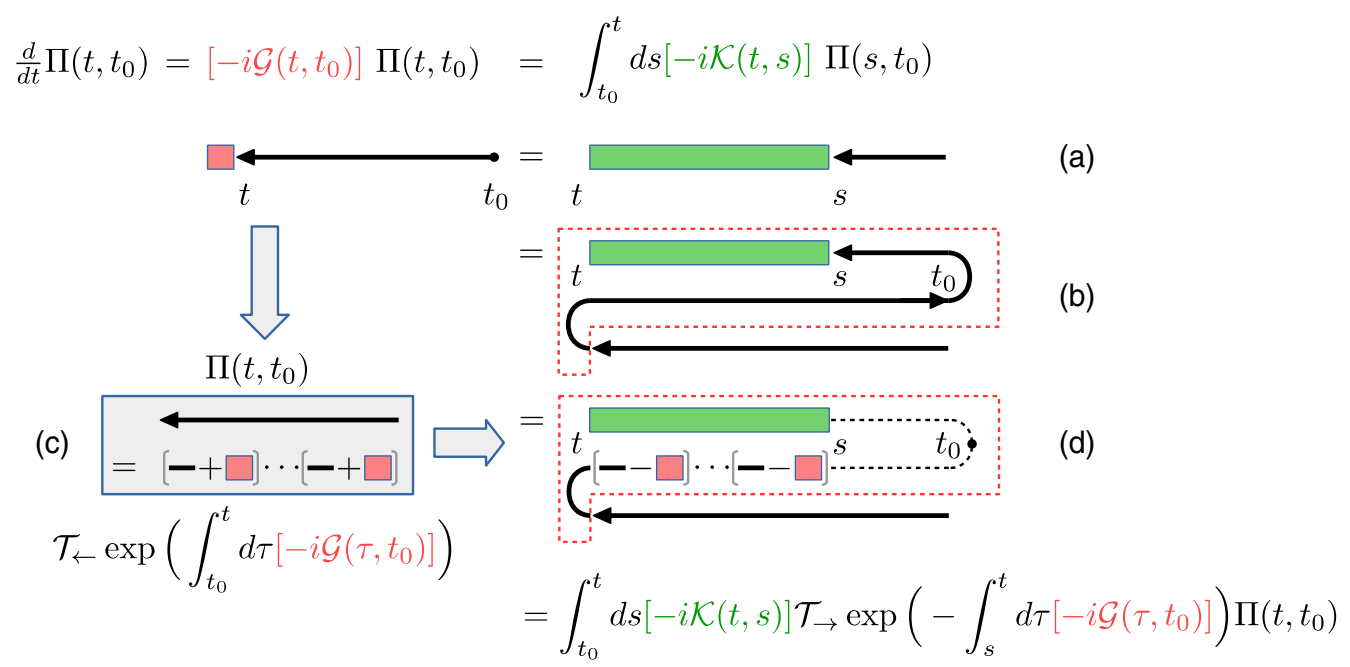

FIG. 1. Graphical representation of the derivation of the functional fixed-point equation (10). (a) Equivalent expressions for $d \Pi\left(t, t_{0}\right) / d t$ as given by the two QMEs. (b) Insertion of canceling backward and forward propagation to initial time $t_{0}$. (c) Evolution $\mathcal{T}_{\leftarrow} \exp \left\{\int_{t_{0}}^{t} d \tau\left[-i \mathcal{G}\left(\tau, t_{0}\right)\right]\right\}=\lim _{N \rightarrow \infty}\left[\mathcal{I}-i \mathcal{G}\left(t_{1}\right) \Delta t_{1}\right] \ldots\left[\mathcal{I}-i \mathcal{G}\left(t_{N}\right) \Delta t_{N}\right]$ expressed as product of infinitesimal steps for the sake of illustration. (d) Backward propagation to memory time $s$ expressed in terms of $\mathcal{G}$ using the divisor. The self-consistency expressed by the functional fixed-point equation (10) arises from backward propagation that is needed to enforce the time-local structure of QME (2) onto the QME (1). For time-translational systems in the stationary limit the generator becomes $\mathcal{G}\left(\tau, t_{0}\right) \rightarrow \mathcal{G}(\infty)$ and literally takes on the role of the complex frequency at which $\hat{\mathcal{K}}(\omega)$ is sampled in Eq. (15).

We already note that the functional $\hat{\mathcal{K}}[X]$ may have fixed points other than $X=\mathcal{G}$. The nonuniqueness and stability of fixed points are further discussed in Sec. V for two specific models.

In Fig. 1 we graphically outline this derivation. This highlights that time-local propagation with $\mathcal{G}$ needs to be consistent with time-locally evolving backward with $\mathcal{G}$ and time-nonlocally propagating forward with the memory kernel. We stress that Eq. (10) is a transformation between two complementary descriptions of the same dynamics. It thus also applies to approximate dynamics $\Pi^{\prime}$ generated equivalently by some $\mathcal{K}^{\prime}$ and $\mathcal{G}^{\prime}$, and thus has broad applicability. In the present paper we aim to highlight the intrinsic functioning of the fixed-point relation and therefore focus on its implications for exactly solvable dynamics.

Equation (10) is explicitly consistent with trace preservation, a fundamental property of the dynamics. Because of the ordering in Eq. (11), where the kernel $\mathcal{K}$ stands to the left of the exponential, the trace-preservation property of the kernel, $\operatorname{Tr} \mathcal{K}(t, s) \bullet=0$, implies the corresponding property of the generator, $\operatorname{Tr} \mathcal{G}\left(t, t_{0}\right) \bullet=0$, where $\bullet$ denotes some operator argument. In fact, for any superoperator function $X\left(t, t_{0}\right)$, one has

$$
\operatorname{Tr} \hat{\mathcal{K}}[X]\left(t, t_{0}\right)=0 .
$$

Moreover, the connection between the Hermicitypreservation property of the kernel and the generator can also be easily checked: Since $-i \mathcal{K}(t) A=\left[-i \mathcal{K}(t) A^{\dagger}\right]^{\dagger}=$ $\mathcal{H}[-i \mathcal{K}(t)] \mathcal{H} A$ for any operator $A$, where $\mathcal{H} A:=A^{\dagger}$ is an antilinear superoperator, we have

$$
\mathcal{H}\left\{-i \hat{\mathcal{K}}[X]\left(t, t_{0}\right)\right\} \mathcal{H}=-i \hat{\mathcal{K}}[-\mathcal{H} X \mathcal{H}]\left(t, t_{0}\right)
$$

\section{STATIONARY FIXED-POINT EQUATION}

We now focus on the implications for time-translational systems in the stationary limit and consider the case where the generator converges to a constant superoperator $\mathcal{G}(\infty)=\lim _{t_{0} \rightarrow-\infty} \mathcal{G}\left(t-t_{0}\right)$. Then the idea is that at large $t-t_{0}$ we can replace [107] the time-ordered exponential in Eq. (11) by an exponential function:

$$
\begin{aligned}
\hat{\mathcal{K}}[\mathcal{G}]\left(t-t_{0}\right) & =\int_{t_{0}}^{t} d s \mathcal{K}(t-s) \mathcal{T}_{\rightarrow} e^{i \int_{s}^{t} d \tau \mathcal{G}\left(\tau-t_{0}\right)} \\
& \approx \int_{-\infty}^{t} d s \mathcal{K}(t-s) e^{i(t-s) \mathcal{G}(\infty)}
\end{aligned}
$$

Here we use that typically either the generator has already become stationary, $\mathcal{G}\left(\tau-t_{0}\right) \approx \mathcal{G}(\infty)\left(\tau \geq s \gg t_{0}\right)$, or the memory kernel has already decayed $(t \gg s)$, thus suppressing the expression. Hence we obtain the stationary fixed-point equation:

$$
\mathcal{G}(\infty)=\hat{\mathcal{K}}(\mathcal{G}(\infty))
$$

It features instead of Eq. (11) the much simpler extension of the Laplace transform (4) with frequency $\omega$ replaced by the time-constant superoperator $X$ :

$$
\hat{\mathcal{K}}(X)=\int_{0}^{\infty} d s \mathcal{K}(s) e^{i s X} .
$$




\section{A. Exact sampling relation between spectral decompositions}

The stationary fixed-point equation (15) immediately makes clear that in general the stationary generator $\mathcal{G}(\infty)$ is not the low-frequency limit of the memory kernel, $\hat{\mathcal{K}}(0)=\lim _{\omega \rightarrow i 0^{+}} \hat{\mathcal{K}}(\omega)$. We now make precise which parts of the frequency dependence of the memory kernel $\hat{\mathcal{K}}(\omega)$ matter in the stationary limit. To this end, assume that one can diagonalize the stationary generator, $\mathcal{G}(\infty)=$ $\left.\sum_{i} g_{i} \mid g_{i}\right)\left(\bar{g}_{i} \mid\right.$, and denote the distinct left and right eigenvectors to the same eigenvalue $g_{i}$ by $\left(\bar{g}_{i} \mid\right.$ and $\left.\mid g_{i}\right)$, respectively, which satisfy the Hilbert-Schmidt [108] biorthogonality relation $\left(\bar{g}_{i} \mid g_{i^{\prime}}\right)=\delta_{i i^{\prime}}$. Insertion into Eq. (15) gives $\left.\mathcal{G}(\infty)=\sum_{i} \hat{\mathcal{K}}\left(g_{i}\right) \mid g_{i}\right)\left(\bar{g}_{i} \mid\right.$ with the ordinary Laplace transform (4) evaluated at $\omega=g_{i}$. Focusing on nondegenerate eigenvalues, we therefore have

$$
\left.\left.\left.\hat{\mathcal{K}}\left(g_{i}\right) \mid g_{i}\right)=\mathcal{G}(\infty) \mid g_{i}\right)=g_{i} \mid g_{i}\right) .
$$

Diagonalizing the kernel after Laplace transforming, $\left.\hat{\mathcal{K}}(\omega)=\sum_{j} k_{j}(\omega) \mid k_{j}(\omega)\right)\left(\bar{k}_{j}(\omega) \mid\right.$, implies that at designated frequencies $\omega=g_{i}$ one of its eigenvalues, labeled $j=f_{i}$, must coincide with an eigenvalue $g_{i}$ of the stationary generator $\mathcal{G}(\infty)$ :

$$
k_{f_{i}}\left(g_{i}\right)=g_{i} .
$$

The right eigenvectors can then be normalized to coincide:

$$
\left.\left.\mid k_{f_{i}}\left(g_{i}\right)\right)=\mid g_{i}\right) .
$$

Importantly, the eigenvectors of the kernel $\left.\mid k_{j}(\omega)\right)$ can also contain poles, which have an important impact on the evolution, as illustrated explicitly in Sec. V B. However since $\mathcal{G}(\infty)$ was assumed to be finite, it cannot sample any of these eigenvector poles of the kernel.

We note that the left eigenvectors $\left(\bar{g}_{i} \mid\right.$ and $\left(\bar{k}_{f_{i}}\left(g_{i}\right) \mid\right.$ in general differ with one important exception, labeled by $i=0$ : From the trace-preservation property of the dynamics [see Eq. (12)] it follows that both $\mathcal{G}(\infty)$ and $\hat{\mathcal{K}}(\omega)$ at every frequency $\omega$ have the left zero eigenvector $(\mathbb{1} \mid=\operatorname{Tr} \bullet$, the trace functional. The corresponding zero eigenvalue is denoted by $g_{0}=k_{0}(\omega)=0$ for all $\omega$ labeling $f_{0}=0$. Thus, a nontrivial consequence of Eq. (17) is that the associated right zero eigenvectors of $\mathcal{G}(\infty)$ and $\hat{\mathcal{K}}(0)$, respectively, coincide with the stationary state:

$$
\left.\left.\left.\mid g_{0}\right)=\mid k_{0}(0)\right)=\mid \rho(\infty)\right) .
$$

This generalizes the result of Ref. [35], which proved this statement for probability vectors evolving with a time-local master equation (i.e., for probabilities only).

We summarize the key result of this section: For Hilbertspace dimension $d$ the stationary time-local generator, with its finite set of eigenvalues $g_{0}, \ldots, g_{d^{2}-1}$, can be written as

$$
\left.\mathcal{G}(\infty)=\sum_{i} k_{f_{i}}\left(g_{i}\right) \mid k_{f_{i}}\left(g_{i}\right)\right)\left(\bar{g}_{i} \mid\right.
$$

It samples one term of the Laplace-transformed memory kernel at each of the frequencies $\omega=g_{0}, \ldots, g_{d^{2}-1}$ :

$$
\begin{aligned}
\hat{\mathcal{K}}\left(g_{i}\right)= & \left.k_{f_{i}}\left(g_{i}\right) \mid k_{f_{i}}\left(g_{i}\right)\right)\left(\bar{k}_{f_{i}}\left(g_{i}\right) \mid\right. \\
& \left.+\sum_{j \neq f_{i}} k_{j}\left(g_{i}\right) \mid k_{j}\left(g_{i}\right)\right)\left(\bar{k}_{j}\left(g_{i}\right) \mid .\right.
\end{aligned}
$$

From each sampled frequency only a single right eigenvector $\left.\mid k_{f_{i}}\left(g_{i}\right)\right)$ for one specific eigenvalue satisfying $k_{f_{i}}\left(g_{i}\right)=g_{i}$ is needed to construct $\mathcal{G}(\infty)$. Importantly, its left eigenvectors $\left(\bar{g}_{i} \mid\right.$ are determined by the right ones through the biorthogonality constraint.

Anticipating later discussion, we note that some intuitive ideas turn out to be incorrect. First, the sampling formula shows that in general nonzero frequencies of $\hat{\mathcal{K}}(\omega)$ may matter at stationarity. It thus makes precise that "memory," often understood as retardation or frequency dependence of the kernel $[28,37,38,84,85]$, is in general not the same as memory defined by a Markovian semigroup $[12,13,39,41,52]$, in which $\mathcal{G}(\infty)$ naturally appears, as we discuss later. Second, the sampled frequencies $g_{i}$ need not be the eigenvalues with the smallest decay rates $\left[-\operatorname{Im} k_{j}\left(\omega_{p}\right)\right]$, as illustrated in Sec. V.

The sampling formula (21) implies that the analytical calculation of the typically more complicated quantity $\mathcal{G}(\infty)$ can in principle be reduced to the calculation of $\hat{\mathcal{K}}(\omega)$ at just $d^{2}$ specific frequencies. We show in Sec. IV A how $\mathcal{G}(\infty)$ can be iteratively computed from $\hat{\mathcal{K}}(\omega)$, thus determining which frequencies are actually sampled. It is therefore not necessary to compute the transient generator $\mathcal{G}(t)$ in order to compute $\mathcal{G}(\infty)$. This is a significant advance since $\hat{\mathcal{K}}$ can be approximated accurately for complicated many-body dynamics using well-developed techniques [20,21,24,26,27]. As mentioned, our relations remain valid when dealing with such approximate kernels: they are simply a way to change from a time-nonlocal to a time-local representation.

\section{B. Exact time-evolution poles}

We now compare the sampling relation (21) with the formal exact solution for time-translational systems obtained by the resolvent method: Laplace transforming the time-nonlocal QME (1) to obtain the "Green's function" or resolvent $\hat{\Pi}(\omega)=i /[\omega-\hat{\mathcal{K}}(\omega)]$, and transforming back by integration along a clockwise oriented contour $\mathcal{C}$ closed in the lower half of the complex plane, we get: 


$$
\begin{aligned}
\Pi\left(t-t_{0}\right)= & \int_{\mathcal{C}} \frac{d \omega}{2 \pi} \hat{\Pi}(\omega) e^{-i \omega\left(t-t_{0}\right)} \\
= & -i \sum_{p} \operatorname{Res}\left[\hat{\Pi}\left(\omega_{p}\right) e^{-i \omega_{p}\left(t-t_{0}\right)}\right] \\
& +\int_{\mathrm{bc}} \frac{d \omega}{2 \pi} \hat{\Pi}(\omega) e^{-i \omega\left(t-t_{0}\right)}
\end{aligned}
$$

Here $\operatorname{Res}\left[f\left(\omega_{p}\right)\right]$ is the residue at pole $\omega_{p}$ and "bc" indicates integration over possible branch cut contributions of $\hat{\Pi}(\omega)$; see Refs. [20,21,24,109] for details and applications. The eigenvalue poles of $\hat{\Pi}(\omega)$ solve the equation $\omega_{p}=k_{j}\left(\omega_{p}\right)$ for some eigenvalue of $\hat{\mathcal{K}}$. By our result (18) the eigenvalues of $\mathcal{G}(\infty)$ are guaranteed to be included among these eigenvalue poles of $\hat{\Pi}(\omega)$. Thus, our stationary fixed-point equation (15) reveals how the time-local approach keeps track of these characteristic frequencies of the evolution, which are explicit in the time-nonlocal approach.

In other words, for time-translational systems the relation $\mathcal{G}(\infty)=\hat{\mathcal{K}}(\mathcal{G}(\infty))$ establishes that the time-local generator $\mathcal{G}(\infty)$ is a superoperator-valued characteristic "frequency" of the evolution. To be sure, there are further contributions from nonsampled poles and branch cuts, which can be infinitely many and may also involve the eigenvectors [21]. These are encoded in the transient fixedpoint equation (10) through the anti-time-ordered integration (11). Thus, the eigenvalues of $\mathcal{G}(\infty)$ generally do not exhaust all the eigenvalue poles of $\hat{\Pi}(\omega)$. Which of the eigenvalues of $\hat{\mathcal{K}}(\omega)$ satisfying $\omega_{p}=k_{j}\left(\omega_{p}\right)$ are eigenvalues of $\mathcal{G}(\infty)$ is not a priori clear, as discussed above.

Our result (21a) now reveals that the first contribution to the exact dynamics (22) actually contains a Markovian semigroup exponential:

$$
\Pi\left(t-t_{0}\right)=e^{-i\left(t-t_{0}\right) \mathcal{G}(\infty)} \mathcal{S}+\cdots,
$$

where center dots denote the abovementioned nonsampled contributions. If $\mathcal{G}(\infty)$ exists, one might expect that the evolution for long times will eventually follow this semigroup dynamics. However, this exponential term is already modified by the time-constant superoperator,

$$
\left.\mathcal{S}=\sum_{i} \frac{1}{1-\frac{\partial k_{f_{i}}}{\partial \omega}\left(g_{i}\right)} \mid g_{i}\right)\left(\bar{k}_{f_{i}}\left(g_{i}\right) \mid\right.
$$

obtained from the residues [110] in Eq. (22) using Eq. (19). The superoperator $\mathcal{S}$ is of practical importance as it relates to the so-called slippage of the initial condition, a wellknown procedure for improving Markovian approximations [95-99]; see discussion in Sec. VI.

\section{Nonperturbative semigroup approximations}

We can now address the puzzling issue regarding the more basic approximation strategy that we mentioned in the Introduction: The equivalent QMEs (1) and (2) "naturally" lead to semigroup approximations which differ, even when constructed from the exact $\mathcal{G}$ and $\mathcal{K}$.

Stationary generator $\mathcal{G}(\infty)$.- Assuming that the generator converges to a stationary value $\mathcal{G}(\infty)$ we can try to approximate the time-local QME (2) for large $t$ by replacing the generator by its constant stationary value, $(d / d t) \rho(t) \approx-i \mathcal{G}(\infty) \rho(t)$. This idea underlies Refs. [37,38] and motivated the direct calculation of $\mathcal{G}(\infty)$ by a series expansion in the coupling in Ref. [35]. The resulting approximate dynamics,

$$
\begin{aligned}
\Pi\left(t, t_{0}\right) & \approx e^{-i\left(t-t_{0}\right) \mathcal{G}(\infty)} \\
& \left.=\sum_{i} e^{-i g_{i}\left(t-t_{0}\right)} \mid g_{i}\right)\left(\bar{g}_{i} \mid,\right.
\end{aligned}
$$

has an interesting feature: There are many evolutions for which the asymptotic generator $\mathcal{G}(\infty)$ has a GKSL form $[12,13]$ with non-negative jump rates, which guarantees that the approximation is completely positive in addition to trace preserving. Nonperturbative approximations preserving both these properties are notoriously difficult to construct, especially starting from microscopic models [44,111-113]. Here the class of evolutions goes beyond semigroups by including all CP-divisible evolutions, but also allowing for certain nonCP-divisible ones [114].

Our sampling result (21) allows this to be compared with a corresponding approximation in the Laplace resolvent approach to the time-nonlocal QME: if one keeps the first term of Eq. (22) and selects only the fixed-point poles $\omega=g_{i}$, then one obtains the semigroup approximation together with the initial-slip correction $\mathcal{S}$ as in Eq. (23). Because of the automatic inclusion of $\mathcal{S}$, this approximation is neither a semigroup nor a CP map around the initial time $t_{0}$ [94]. This may give faster convergence but also fail dramatically; see discussion in Sec. VI. In contrast, the semigroup approximation (25) does not suffer from such problems.

Low-frequency memory kernel $\hat{\mathcal{K}}(0)$. - - Starting instead from the time-nonlocal QME (1), one may argue that for slowly varying dynamics only the low-frequency part of the memory kernel matters. Replacing $\rho(s) \rightarrow \rho(t)$ in the integrand and taking $t_{0} \rightarrow-\infty$, one then obtains $\dot{\rho}(t) \approx$ $-i \hat{\mathcal{K}}(0) \rho(t)$ with the approximate solution:

$$
\begin{aligned}
\Pi\left(t, t_{0}\right) & \approx e^{-i\left(t-t_{0}\right) \hat{\mathcal{K}}(0)} \\
& \left.=\sum_{j} e^{-i k_{j}(0)\left(t-t_{0}\right)} \mid k_{j}(0)\right)\left(\bar{k}_{j}(0) \mid .\right.
\end{aligned}
$$

In the resolvent approach this approximation is equivalent to neglecting all frequency dependence of the memory 
kernel, $\hat{\Pi}(\omega) \approx i /[\omega-\hat{\mathcal{K}}(0)]$, leaving only $d^{2}$ eigenvalue poles $\omega_{j}=k_{j}(0)$. In contrast to the case of $\mathcal{G}(\infty)$ we know of no general conditions that guarantee that $\hat{\mathcal{K}}(0)$ generates a completely positive evolution for some broad class of nontrivial models. Even when it is known that $\mathcal{G}(\infty)$ has non-negative GKSL coefficients-ensuring Eq. (25) is completely positive - one still has to explicitly check that the same holds for $\hat{\mathcal{K}}(0)$. Although both approximations (25) and (26) nonperturbatively account for oscillation frequencies and decay rates in a different way, it follows from the sampling result (19) that both converge to the exact stationary state. In Sec. V we illustrate their difference. Note, however, that $\mathcal{G}(\infty)=\hat{\mathcal{K}}(0)$ is possible also for a nonsemigroup evolution [Eq. (53)].

\section{Summing the memory expansion}

Whereas the argument leading to Eq. (26) may be justified in the weak coupling limit, it has been noted that when computing $\hat{\mathcal{K}}$ to higher order in the systemenvironment coupling this becomes inconsistent [36-38, 116]. In terms of Eq. (8) this means that one must not only expand the kernel $\mathcal{K}(t-s)$ in the memory time $s$ relative to the current time $t$, but simultaneously expand $\Pi\left(s, t_{0}\right)=$ $\Pi\left(t, t_{0}\right)-(t-s) \partial \Pi\left(t, t_{0}\right) / \partial t+\cdots$ under the memory integral. This way Ref. [37] obtained a stationary timelocal QME with an approximate generator:

$$
\mathcal{G}(\infty) \approx \hat{\mathcal{K}}(0)+\frac{\partial \hat{\mathcal{K}}}{\partial \omega}(0) \hat{\mathcal{K}}(0) .
$$

When computing $\hat{\mathcal{K}}(0)$ to second order in, e.g., a tunnel coupling, the first-order contributions to the second term are comparable [37] and may lead to cancellations that are necessary to respect complete positivity $[84,85]$.

One may roughly understand Eq. (27) as follows: to obtain $\mathcal{G}(\infty)$ one linearizes the frequency dependence of the memory kernel $\hat{\mathcal{K}}(\omega) \approx \hat{\mathcal{K}}(0)+[\partial \hat{\mathcal{K}} / \partial \omega(0)] \omega$ and evaluates it at the characteristic frequency $\omega=\mathcal{G}(\infty) \approx$ $\hat{\mathcal{K}}(0)$ of the system, which in first approximation is the lowfrequency kernel itself. This tentative picture is made rigorous by our fixed-point equation (15), where the frequency is likewise replaced by a superoperator, but in a self-consistent way. In Ref. [38] the approximation (27) was generalized to higher orders by applying partial integrations of the time-nonlocal QME (2), which can be shown to be equivalent to further continuing the memory expansion of Ref. [37]. In the Appendix C we show how this gradient expansion can be expressed in Moyal brackets $[76,77]$ with respect to time similar to that used in Green's function techniques [78,79]. It has also recently been used to combine QMEs with time-dependent density-functional theory $[82,83]$.

Thus, starting from the time-nonlocal QME one is led to a time-local QME by a memory expansion (26). Another key result of this paper is that this series can in fact be summed up to all orders as we show in Appendix B. One finds that the constant generator that accounts for all memory terms of the stationary time-nonlocal QME is the stationary time-local generator obeying $\mathcal{G}(\infty)=$ $\hat{\mathcal{K}}(\mathcal{G}(\infty))$, our stationary fixed-point equation (15). This means that our sampling formula (21) is the nonperturbative result of this memory expansion: The infinite sum of memory terms-featuring all derivatives of $\hat{\mathcal{K}}(\omega)$ at zero frequency - can be condensed into a finite sum of contributions of $\hat{\mathcal{K}}(\omega)$ at just $d^{2}$ finite frequencies $\omega=g_{i}$.

Importantly, the memory expansion can even be summed up for the full transient dynamics, thereby recovering $\mathcal{G}\left(t, t_{0}\right)=\hat{\mathcal{K}}[\mathcal{G}]\left(t, t_{0}\right)$, the functional fixed-point equation (10) (Appendix B). By making use of the divisor we can give a closed formula for terms of arbitrary order [Eqs. (B5) and (B8)]. Altogether, this shows that Eqs. (10) and (15) are very useful for generating gradient expansions in time when given a memory kernel $\mathcal{K}$. We next explore a different approach where one solves for the transient $\mathcal{G}\left(t-t_{0}\right)$, giving approximate evolutions which are not semigroups as Eq. (25) and (26). Also there both $\mathcal{G}(\infty)$ and $\hat{\mathcal{K}}(0)$ play an interesting role.

\section{ITERATIVE CONSTRUCTION OF GENERATOR FROM MEMORY KERNEL}

Our final key result is that the fixed-point equation may be turned into a computational tool to obtain $\mathcal{G}$ from a given memory kernel $\mathcal{K}$ computed using a method of choice. We focus on time-translational systems-setting $t_{0}=0$-and the ideal situation where $\mathcal{K}$ has been computed exactly.

\section{A. Iteration for stationary generator}

The simplest scenario is where one iteratively solves Eq. (15) to find $\mathcal{G}(\infty)$ directly from $\mathcal{K}(t)$ or $\hat{\mathcal{K}}(\omega)$, i.e., without considering the transient evolution $\Pi(t)$ or the transient generator $\mathcal{G}(t)$. Using the converged result one may then set up the nonperturbative semigroup (25) to approximate the full evolution $\Pi(t)$.

First, consider the low-frequency kernel as an initial approximation to the generator, $\mathcal{G}^{(0)}(\infty)=\hat{\mathcal{K}}(0)$, as in Eq. (27). If the exact dynamics is a semigroup, $\mathcal{K}(t)=$ $\hat{\mathcal{K}}(0) \bar{\delta}(t)$ and $\mathcal{G}(t)=\hat{\mathcal{K}}(0)$, then this already is the fixed point since $\mathcal{G}^{(1)}(\infty)=\hat{\mathcal{K}}(\hat{\mathcal{K}}(0))=\hat{\mathcal{K}}(0)$. This may also happen for nonsemigroup evolutions [Eq. (54)]. In general, further approximations are obtained by $n$-fold iteration, $\mathcal{G}^{(n)}(\infty)=\hat{\mathcal{K}}(\ldots \hat{\mathcal{K}}(\hat{\mathcal{K}}(0)))$. Inspecting the first iteration,

$\left.\mathcal{G}^{(1)}(\infty)=\hat{\mathcal{K}}(\hat{\mathcal{K}}(0))=\sum_{j \neq 0} \hat{\mathcal{K}}\left(k_{j}(0)\right) \mid k_{j}(0)\right)\left(\bar{k}_{j}(0) \mid\right.$,

we see that the stationary state $\left.\mid k_{0}(0)\right)$ remains unaffected (trace preservation), but in general all $j \neq 0$ contributions are 
altered by the memory kernel evaluated at finite frequencies, thus generating a difference between $\hat{\mathcal{K}}(0)$ and $\mathcal{G}(\infty)$.

The convergence of this procedure with $n$ is certainly not obvious, but our first applications in Sec. V are encouraging. Indeed, one can consider starting the iteration from any initial superoperator $\mathcal{G}^{(0)}(\infty)=X$. In this case, property (12) guarantees that the iteration trajectory $\mathcal{G}^{(n)}(\infty)=$ $\hat{\mathcal{K}}(\ldots \hat{\mathcal{K}}(X))$ is confined to the linear space of tracepreserving superoperators irrespective of $X$. If $i X$ is Hermicity preserving, then the trajectory will additionally be confined to such superoperators by property (13).

\section{B. Functional iteration for transient generator}

We next describe the more complicated iteration of the functional equation (10). Here the aim is to construct the full transient generator $\mathcal{G}(t)$ starting from the memory kernel $\mathcal{K}(t)$. As a preparation we decompose the kernel into its time-local ( $\bar{\delta}$-singular) part and a remaining timenonlocal part:

$$
\mathcal{K}(t)=\mathcal{K}_{L} \bar{\delta}(t)+\mathcal{K}_{N}(t) .
$$

In addition to the system Liouvillian $L$, the part $\mathcal{K}_{L}$ may contain an environment-induced contribution (as for fermionic wideband models $[7,8,93]$ as studied in Sec. V B), but this need not be the case (as in the model studied in Sec. VA). Inserting Eq. (29) into the functional (11) we obtain

$\mathcal{G}^{(n+1)}(t)=\mathcal{K}_{L}+\int_{0}^{t} d s \mathcal{K}_{N}(t-s) \mathcal{T}_{\rightarrow} e^{i \int_{s}^{t} d \tau \mathcal{G}^{(n)}(\tau)}$.

Iterating this equation starting from the constant function $\mathcal{G}^{(0)}(t)=\hat{\mathcal{K}}(0)$ gives approximations $\mathcal{G}^{(n)}(t)$ which generate evolutions with two important properties at every iteration.

First, each approximation is accurate at long times, provided $\mathcal{G}(t)$ has a stationary limit and Eq. (10) converges to Eq. (15). Our choice of starting point ensures by Eq. (20) that $\left.\mathcal{G}^{(n)}(t) \mid \rho(\infty)\right)=0$ holds initially for $n=0$, implying that the generated evolution goes to the exact stationary state for $t \rightarrow \infty$. Arguing as in Eq. (14) we find that this also holds for the next iteration: $\left.\mathcal{G}^{(n+1)}(\infty) \mid \rho(\infty)\right)=$ $\left.\left.\lim _{t \rightarrow \infty}\left[\mathcal{K}_{L}+\int_{0}^{t} d s \mathcal{K}_{N}(t-s)\right] \mid \rho(\infty)\right)=\hat{\mathcal{K}}(0) \mid \rho(\infty)\right)=0$. The same argument also applies for starting point $\mathcal{G}^{(0)}(t)=$ $\mathcal{G}(\infty)$ [Eq. (20)] or any starting point $X$ for which $X \mid \rho(\infty))=0$. However, starting from the memory kernel formalism, $\hat{\mathcal{K}}(0)$ is already available.

Second, each generated approximation is also accurate at short times. To see this, note that at the initial time the generator is given by the time-local part of the kernel,

$$
\mathcal{G}(0)=\mathcal{K}_{L},
$$

which we split off from the generator,

$$
\mathcal{G}(t)=\mathcal{K}_{L}+\mathcal{G}_{N}(t), \quad \mathcal{G}_{N}(0)=0 .
$$

The second term incorporates all effects due to the timenonlocal part of the kernel $\mathcal{K}_{N}(t)$. For the first iteration we have

$$
\begin{aligned}
\mathcal{G}^{(1)}(t) & =\mathcal{K}_{L}+\int_{0}^{t} d s \mathcal{K}_{N}(t-s) e^{i \hat{\mathcal{K}}(0)(t-s)} \\
& \approx \mathcal{K}_{L}+t \mathcal{K}_{N}(0)+\cdots
\end{aligned}
$$

as dictated by the short-time limit of the time-nonlocal part of the memory kernel. This implies that in the exponential of the next iteration we similarly have at short times $\int_{s}^{t} d \tau \mathcal{G}^{(1)}(\tau) \approx(t-s) \mathcal{K}_{L}$, giving the same leading behavior. Thus, each iteration $n \geq 1$ coincides with the exact initial generator (31) including the linear order, $\mathcal{G}^{(n)}(t)=\mathcal{K}_{L}+$ $t \mathcal{K}_{N}(0)+\cdots$. Clearly, no semigroup approximation can achieve this.

The convergence of this iteration is again not evident and an analysis of the local stability is complicated due to the time nonlocality of the superoperator equations. Remarkably, we numerically find for several models that this procedure can be made to work, even when the generator is time singular (Sec. VA) or has time-dependent algebraic structure (Sec. V B).

\section{EXAMPLES}

\section{A. Dissipative Jaynes-Cummings model}

We first illustrate our findings for the dissipative JaynesCummings model $[6,17,100,101]$, which is algebraically simple but can show challenging time singularities in the generator. This exactly solvable model describes a twolevel atom with transition frequency $\varepsilon\left(H=\varepsilon d^{\dagger} d\right.$ with $\left\{d, d^{\dagger}\right\}=\mathbb{1}$ ) interacting with a continuous bosonic reservoir $\left[H_{R}=\int d \omega \omega b_{\omega}^{\dagger} b_{\omega}\right.$ with $\left.\left[b_{\omega}, b_{\omega^{\prime}}^{\dagger}\right]=\delta\left(\omega-\omega^{\prime}\right) \mathbb{1}\right]$ initially in a vacuum state $|0\rangle$. The coupling is bilinear,

$$
H_{T}=\int d \omega \sqrt{\frac{\Gamma(\omega)}{2 \pi}}\left(d^{\dagger} b_{\omega}+b_{\omega}^{\dagger} d\right),
$$

with real amplitudes set by a spectral density $\Gamma(\omega)$. The occupation numbers of reservoir modes are either 0 or 1 due to a dynamical constraint: the coupling (34) conserves the total excitation number $d^{\dagger} d+\int d \omega b_{\omega}^{\dagger} b_{\omega}$. Here we study the effects of energy-dependent coupling $\Gamma(\omega)$ without initial reservoir statistics $(T=0)$ : We assume a Lorentzian profile of width $\gamma$ whose maximum value $\Gamma \equiv \Gamma(\varepsilon)$ lies precisely at the atomic resonance:

$$
\Gamma(\omega)=\Gamma \frac{\gamma^{2}}{(\varepsilon-\omega)^{2}+\gamma^{2}} .
$$


Although this model has been studied in detail $[17,18,100,101]$ and featured in textbooks [6], the remarkable relation between its generator $\mathcal{G}$ and memory kernel $\mathcal{K}$ has not been noted, but see Ref. [75]. All results below can be generalized to any profile $\Gamma(\omega)$.

From the solution [6] of the total-system state $\left|\psi_{\text {tot }}(t)\right\rangle$, with $\left|\psi_{\text {tot }}(0)\right\rangle=|\psi(0)\rangle \otimes|0\rangle$, we extract the propagator $\operatorname{Tr}_{R}\left\{\left|\psi_{\text {tot }}(t)\right\rangle\left\langle\psi_{\text {tot }}(t)\right|\right\}=\Pi(t)|\psi(0)\rangle\langle\psi(0)|$ working in the Schrödinger picture and setting $t_{0}=0$. It has the form of an amplitude damping channel [117] with spectral decomposition

$$
\begin{aligned}
\Pi(t)= & \mid 00)\left[\left(\left.00|+(11 \mid]+| \pi(t)\right|^{2}[\mid 11)-\mid 00\right)\right](11 \mid \\
& +\pi(t) \mid 01)\left(01\left|+\pi(t)^{*}\right| 10\right)(10 \mid
\end{aligned}
$$

using $\left.\mid \nu \nu^{\prime}\right)=|\nu\rangle\left\langle\nu^{\prime}\right|$ and $\left(\nu \nu^{\prime} \mid=\left\langle\nu|\bullet| \nu^{\prime}\right\rangle\right.$, where $|\nu\rangle$ denotes the atomic state $\nu=0,1$. The time-dependent parameter reads

$$
\pi(t) \equiv e^{-i \varepsilon t} e^{-\gamma t / 2}\left[\cosh \left(\frac{\gamma^{\prime} t}{2}\right)+\frac{\gamma}{\gamma^{\prime}} \sinh \left(\frac{\gamma^{\prime} t}{2}\right)\right],
$$

where $\gamma^{\prime}:=\sqrt{\gamma(\gamma-2 \Gamma)}$. Thus, an initially excited state evolves with probability $\langle 1|\rho(t)| 1\rangle=|\pi(t)|^{2}$. In the frequency domain we have

$$
\begin{aligned}
\hat{\Pi}(\omega)= & \left.\frac{i}{\omega} \mid 00\right)\left[\left(00\left|+(11 \mid]+\widehat{|\pi|^{2}}(\omega)[\mid 11)-\right| 00\right)\right](11 \mid \\
& +\widehat{\pi}(\omega) \mid 01)\left(01\left|+\widehat{\pi^{*}}(\omega)\right| 10\right)(10 \mid .
\end{aligned}
$$

The Laplace transforms,

$$
\begin{aligned}
\mid \widehat{\left.\pi\right|^{2}}(\omega) & =\frac{1}{4} \frac{\left(\gamma / \gamma^{\prime}-1\right)^{2}}{\gamma+\gamma^{\prime}-i \omega}+\frac{1}{4} \frac{\left(\gamma / \gamma^{\prime}+1\right)^{2}}{\gamma-\gamma^{\prime}-i \omega}-\frac{1}{2} \frac{\gamma^{2} / \gamma^{\prime 2}-1}{\gamma-i \omega}, \\
\hat{\pi}(\omega) & =\frac{\gamma / \gamma^{\prime}+1}{\gamma-\gamma^{\prime}-2 i(\omega-\varepsilon)}-\frac{\gamma / \gamma^{\prime}-1}{\gamma+\gamma^{\prime}-2 i(\omega-\varepsilon)},
\end{aligned}
$$

and $\widehat{\pi^{*}}(\omega)=\left[\hat{\pi}\left(-\omega^{*}\right)\right]^{*}$ determine the finite number of poles of the propagator $\hat{\Pi}(\omega)$ listed in Table I.

It is now straightforward $[17,18]$ to determine the generator $\mathcal{G}(t)=i \dot{\Pi}(t) \Pi^{-1}(t)$ and the kernel $\hat{\mathcal{K}}(\omega)=$ $\omega \mathcal{I}-i \hat{\Pi}^{-1}(\omega)$ whose relation has our interest. The spectral decomposition for the generator reads

$$
\begin{aligned}
\mathcal{G}(t)= & \left.\left.2 i \operatorname{Re}\left(\frac{\dot{\pi}(t)}{\pi(t)}\right)[\mid 11)-\mid 00\right)\right](11 \mid \\
& \left.+i \frac{\dot{\pi}(t)}{\pi(t)} \mid 01\right)\left(01\left|+i\left(\frac{\dot{\pi}(t)}{\pi(t)}\right)^{*}\right| 10\right)(10 \mid,
\end{aligned}
$$

whereas for the kernel in the frequency domain it is
TABLE I. Jaynes-Cummings model. Poles of $\hat{\Pi}(\omega)$ and eigenvalues of $\mathcal{G}(\infty)$ using the abbreviation $\gamma^{\prime}=\sqrt{\gamma(\gamma-2 \Gamma)}$.

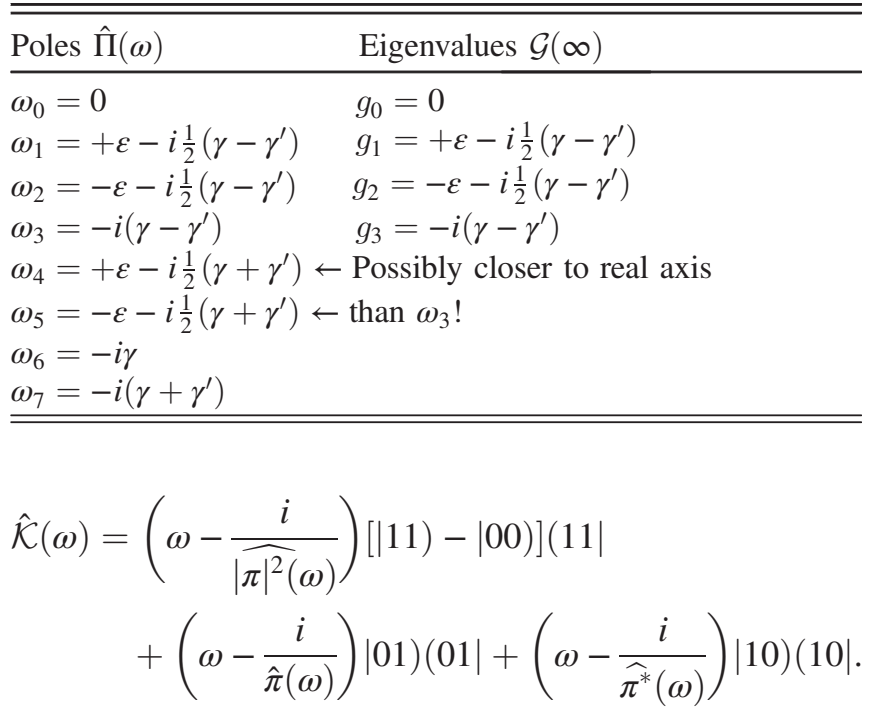

The eigenvalues of $\hat{\mathcal{K}}$ satisfying $k_{j}\left(\omega_{p}\right)=\omega_{p}$ for some $j$ correspond to the poles of $\hat{\Pi}(\omega)$ in Table I.

\section{Overdamped dynamics $(\gamma \geq 2 \Gamma)$}

Even with all explicit expressions in hand, it is by no means obvious that this model obeys our sampling result (21) in the stationary limit $t \rightarrow \infty$. We now first verify this noting that our assumption that $\mathcal{G}(\infty)$ exists holds only for broad spectral densities such that $\gamma \geq 2 \Gamma$. In this case the real quantity $\gamma^{\prime}=\sqrt{\gamma(\gamma-2 \Gamma)} \leq \gamma$ represents a suppression or enhancement of the decay rates $-\operatorname{Im} \omega_{p}$ relative to the value $\gamma$ in Table I. In this overdamped regime, $\lim _{t \rightarrow \infty} \dot{\pi}(t) / \pi(t)=-\frac{1}{2}\left(\gamma-\gamma^{\prime}\right)-i \varepsilon$ converges and the dynamics is CP divisible [118].

Table I shows that the resulting four eigenvalues of $\mathcal{G}(\infty)$ indeed coincide with four of the eight poles of $\hat{\Pi}(\omega)$ as predicted by Eq. (18). Interestingly, $\mathcal{G}(\infty)$ does not always sample the "slowest" part of the evolution, i.e., the poles with the smallest decay rates, even in this simple model. Whereas this happens for sufficiently large broadening $\gamma>\frac{9}{4} \Gamma$, just before entering the underdamped regime there is a range $2 \Gamma<\gamma<\frac{9}{4} \Gamma$, where two nonsampled poles $\omega_{4,5}$ have smaller decay rates than the sampled pole $\omega_{3}$; see Table I. Thus, $\mathcal{G}(\infty)$ is completely determined by the sampling of $\hat{\mathcal{K}}(\omega)$ as dictated by Eq. (21a). This does not illustrate the full complexity of the sampling since the right eigenvectors of $\hat{\mathcal{K}}(\omega)$ are frequency independent and thus trivially provide the right eigenvectors (19) of $\mathcal{G}(\infty)$.

Numerical implementation of the stationary iteration described in Sec. IVA converges in a few steps to the exact stationary generator, which explicitly reads 


$$
\begin{aligned}
-i \mathcal{G}(\infty)= & \left.\left.-\frac{2 \Gamma}{1+\sqrt{1-2 \Gamma / \gamma}}[\mid 11)-\mid 00\right)\right](11 \mid \\
& \left.-\left(+i \varepsilon+\frac{\Gamma}{1+\sqrt{1-2 \Gamma / \gamma}}\right) \mid 01\right)(01 \mid \\
& \left.-\left(-i \varepsilon+\frac{\Gamma}{1+\sqrt{1-2 \Gamma / \gamma}}\right) \mid 10\right)(10 \mid .
\end{aligned}
$$

Importantly, we numerically observe this convergence starting from random initial superoperators $X$. Although other fixed points of Eq. (16) can be constructed [119], we always find that $\mathcal{G}(\infty)$ is the only stable one. Because of this remarkable fact, the iterative solution allows one to infer which of the poles are sampled by $\mathcal{G}(\infty)$. As mentioned earlier, this can be used to assist the identification of the sampled poles in analytical calculations, which aim to exploit Eq. (21).

Given the kernel $\hat{\mathcal{K}}(\omega)$, one can thus find $\mathcal{G}(\infty)$ by iteration directly at stationarity, avoiding the transient time dependence of $\mathcal{G}(t)$. We plot the resulting semigroup approximation (25) in Fig. 2(b) and the different semigroup (26), generated by the exact low-frequency kernel,

$$
\begin{aligned}
-i \hat{\mathcal{K}}(0)= & \left.\left.-\frac{\Gamma}{1+\Gamma /(2 \gamma)}[\mid 11)-\mid 00\right)\right](11 \mid \\
& \left.-\left(+i \varepsilon+\frac{\gamma \Gamma}{2(\gamma+i \varepsilon)}\right) \mid 01\right)(01 \mid \\
& \left.-\left(-i \varepsilon+\frac{\gamma \Gamma}{2(\gamma-i \varepsilon)}\right) \mid 10\right)(10 \mid,
\end{aligned}
$$

in Fig. 2(a). The $\hat{\mathcal{K}}(0)$ semigroup crosses the exact solution already at intermediate times to approach it from above, whereas the $\mathcal{G}(\infty)$ semigroup approaches it from below. Indeed, in the overdamped regime the occupation decay rate of Eq. (42) is always larger than that of Eq. (43). As expected, both semigroups have problems with the initial nonlinear time dependence on the scale $\gamma^{-1}$ set by the reservoir bandwidth (35). Only in the wideband limit $\gamma \rightarrow$ $\infty$ the exact evolution is a semigroup, which in this case is generated by $\mathcal{G}(\infty)=\hat{\mathcal{K}}(0)$.

We have also implemented the functional iteration $\mathcal{G}^{(n)}(t)$ for the transient generator explained in Sec. IV B, using Eq. (30) with $\mathcal{K}_{L}=-i[H, \bullet]$ and $H=\varepsilon d^{\dagger} d$. In Figs. 2(a) and 2(b) we additionally show the evolutions generated by the approximate $\mathcal{G}^{(n)}(t)$ starting from the initial function $\mathcal{G}^{(0)}(t)=\hat{\mathcal{K}}(0)$ and $\mathcal{G}(\infty)$, respectively. Like the semigroups, each approximation approaches the exact stationary state at large times. However, contrary to the semigroups, each iteration is also very accurate at short times; see Eq. (33b). These two constraints enforce rapid convergence at intermediate times throughout the overdamped parameter regime: in Figs. 2(a) and 2(b) we did not
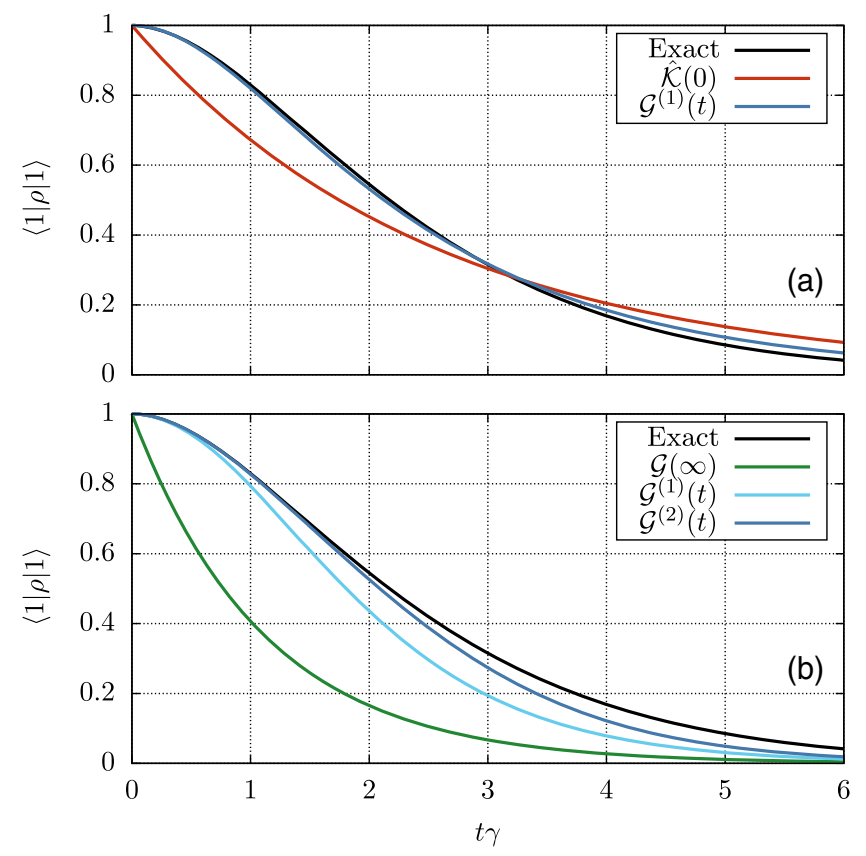

FIG. 2. Jaynes-Cummings model, overdamped regime $\Gamma / \gamma=$ $0.495\left(\gamma^{\prime} / \gamma=0.1\right)$. Decay of the probability $\langle 1|\rho(t)| 1\rangle$ for the excited state when it is initially occupied, $\rho(0)=|1\rangle\langle 1|$. (a) Solutions obtained from the Markovian approximation $\dot{\rho}(t) \approx$ $-i \hat{\mathcal{K}}(0) \rho(t)$ (red line) together with the first iteration $\mathcal{G}^{(1)}$ (cyan line) of the transient fixed-point equation starting from $\mathcal{G}^{(0)}=\hat{\mathcal{K}}(0)$. The exact solution is shown in black. (b) Solutions obtained from the Markovian approximation $\dot{\rho}(t) \approx-i \mathcal{G}(\infty) \rho(t)$ (blue line) together with two iterations of the transient fixed-point equation (30), $\mathcal{G}^{(1)}$ (cyan line) and $\mathcal{G}^{(2)}$ (green line), when started from $\mathcal{G}^{(0)}=\mathcal{G}(\infty)$ and exact solution (black line).

plot the $n=2$ and $n=3$ approximations, respectively, since they are hard to distinguish from the exact solution. Thus, Fig. 2(a) shows that Eq. (33a), based solely on one iteration of the time-nonlocal memory kernel, already provides a remarkably accurate representation of the time-local generator.

\section{Underdamped dynamics $(\gamma<2 \Gamma)$}

For narrow spectral density, $\gamma<2 \Gamma$, the evolution becomes underdamped and nondivisible. The function

$$
\pi(t)=e^{-i \varepsilon t} e^{-\gamma t / 2}\left[\cos \left(\frac{\Omega t}{2}\right)+\frac{\gamma}{\Omega} \sin \left(\frac{\Omega t}{2}\right)\right]
$$

now oscillates with frequency $\Omega \equiv-i \gamma^{\prime}=\sqrt{\gamma(2 \Gamma-\gamma)}$ with roots located at $t_{n}=\frac{2 \pi}{\Omega}[n-(1 / \pi) \arctan (\Omega / \gamma)]$. This qualitative change of $\pi(t)$ has two consequences.

First, the time-local generator $\mathcal{G}(t)$ by itself exhibits singularities as function of time for every $t=t_{n}$ [Eq. (40)]. These dynamics with singular generators have recently received renewed attention [104-106], even though they 
were noted long ago [6]. Importantly these singularities are not spurious, noting that the product $\mathcal{G}(t) \Pi(t)$ remains finite even at $t=t_{n}$. In fact, they are physically meaningful: by identifying the divergent matrix elements of $\mathcal{G}(t)$ one can already infer at which times the solution of the JaynesCummings model will be an entanglement breaking map [120-123], $\left.\Pi\left(t_{n}\right)=\mid 00\right)(\mathbb{1}|=| 0\rangle\langle 0| \operatorname{Tr} \bullet$.

A second consequence is that the stationary limit of $\mathcal{G}(t)$ by itself does not exist, even though the stationary propagator does converge, $\left.\lim _{t \rightarrow \infty} \Pi(t)=\mid 00\right)(\mathbb{1} \mid$, and the low-frequency memory kernel $\hat{\mathcal{K}}(0)$ is well defined. Irrespective of how generic both these complications are, they present perhaps the most crucial challenge to any timelocal approach. It is well known, for example, that perturbative calculations of $\mathcal{G}(t)$ cannot venture beyond the first singularity on the time axis [6,124]. In this sense the model presents a worst-case test for both variants of the fixed-point iteration.

The stationary iteration (Sec. IV A) is simply expected to fail since it relies on the convergence of $\mathcal{G}(t)$ for $t \rightarrow \infty$. Nevertheless, it is interesting to explore what happens. Indeed, the stationary iteration for $\mathcal{G}^{(n)}(\infty)$ does not converge anymore with $n$. However, $\mathcal{G}$ is always block diagonal and we observe that the iterations for the generator on the occupation subspace $\mid 00), \mid 11)$ converge to

$$
\left.\left.\lim _{n \rightarrow \infty} \mathcal{G}_{o}^{(n)}(\infty)=-i \gamma[\mid 11)-\mid 00\right)\right](11 \mid,
$$

whereas the generator $\mathcal{G}_{c}^{(n)}$ on the subspace $\left.\left.\mid 01\right), \mid 10\right)$ of the coherences oscillates indefinitely with $n$. In Fig. 3(a) we plot the time evolution of occupations obtained from the semigroup approximation constructed from Eq. (45). In contrast to the semigroup generated by the well-defined $\hat{\mathcal{K}}(0)$, it gives an accurate envelope for the decay of the excited state, even in the strongly underdamped limit, $\gamma \ll$ $\Gamma$ where $\Omega \approx \sqrt{2 \Gamma \gamma} \gg \gamma$.

The converged part of the iteration can in fact be related to a regularization of $\lim _{t \rightarrow \infty} \mathcal{G}(t)$. Noting that

$$
\frac{\dot{\pi}(t)}{\pi(t)}=-i \varepsilon-\frac{1}{2} \gamma-\frac{1}{2} \Omega \tan \left(\frac{1}{2} \Omega t-\arctan \frac{\gamma}{\Omega}\right),
$$

we see that a principal-value time average over one period amounts to replacing $[\dot{\pi}(t) / \pi(t)] \rightarrow-i \varepsilon-\frac{1}{2} \gamma$. This gives a regularized stationary limit for the generator,

$$
\begin{aligned}
\mathcal{G}(\infty)_{\text {reg }}= & -i \gamma[\mid 11)-\mid 00)](11 \mid \\
& \left.-\left(-\varepsilon+\frac{1}{2} i \gamma\right) \mid 01\right)\left(01\left|-\left(\varepsilon+\frac{1}{2} i \gamma\right)\right| 10\right)(10 \mid,
\end{aligned}
$$

which coincides with the numerically converged block (45) of the iteration. The value of the coherence block exposes a
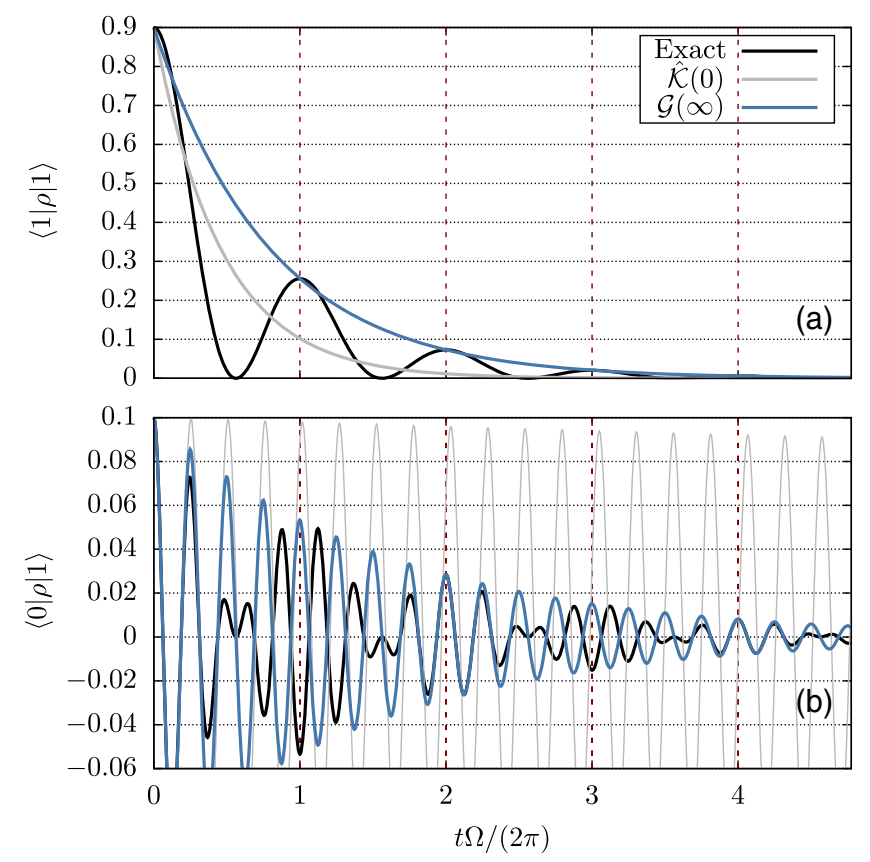

FIG. 3. Jaynes-Cummings model, underdamped regime $\Gamma / \gamma=$ $13(\Omega / \gamma=5)$ and $\varepsilon=20$. (a) Decay of the excited state occupation $\langle 1|\rho(t)| 1\rangle$ and (b) decay of the real part of the coherence $\langle 0|\rho(t)| 1\rangle$. The initial state is $\rho(0)=0.1[\mid 00)+$ $\mid 01)+\mid 10)]+0.9 \mid 11)$. Shown are the solution for the Markovian approximations $\dot{\rho}(t) \approx-i \mathcal{G}(\infty) \rho(t)$ (blue line) with generator obtained by iteration of the stationary fixed point equation (15), and $\dot{\rho}(t) \approx-i \hat{\mathcal{K}}(0) \rho(t)$ (gray line). The exact solution is shown in black.

key complication of the exact evolution of this model. In Fig. 3(b), we show that the semigroup constructed from $\mathcal{G}(\infty)_{\text {reg }}$ describes the decay and oscillation of the coherences accurately in the center of every even time interval. However, it is also accurate up to the sign in every odd interval. The intermediate $\pi$-phase jumps occurring in the exact solution are caused by the divergences of the generator at times $t_{n}$. The stationary fixed-point iteration may thus still be useful beyond the limitations we assumed in the present paper.

Finally, we consider how the transient fixed-point iteration (Sec. IV B) deals with the time singularities in this model. In Fig. 4 we show how the occupations, starting from the semigroup approximation generated by $\hat{\mathcal{K}}(0)$, converge to the exact solution. The first two iterations only improve the solution before the first singularity and even become unphysical at larger times. However, the following iterations also converge beyond the first singularity. The fifth iteration (not shown) is indistinguishable from the exact solution in the shown time interval. More iterations are required to converge the solution in a larger time interval also including the second singularity. The success of our iteration strategy starting from the memory kernel $\mathcal{K}$ highlights its difference to perturbation theory, which 


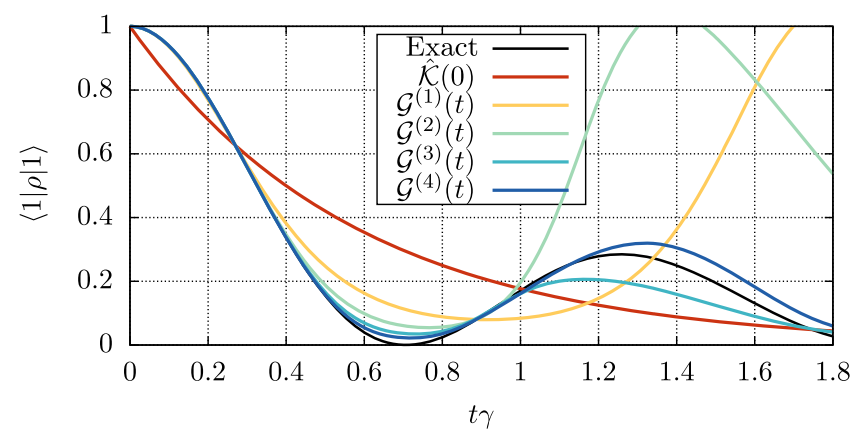

FIG. 4. Jaynes-Cummings model, underdamped regime. Decay of the probability $\langle 1|\rho(t)| 1\rangle$ obtained from generators of the transient iteration for $\Gamma / \gamma=13$ and $\varepsilon=20$ using the starting point $\hat{\mathcal{K}}(0)$.

always fails in capturing dynamics beyond a singularity [6]. Thus even time-singular generators can be locally stable fixed points of the functional $\hat{\mathcal{K}}$. Finally, we note that the fixed-point iteration for the coherences is more challenging, but we do not exclude that this is only a numerical challenge.

\section{B. Finite-temperature resonant level model}

We complement the above by an analysis of the fermionic resonant level model. Although its generator has no time singularities, its time-dependent algebraic structure provides a challenge complementary to the previous model. The Hamiltonian is formally identical to that of the Jaynes-Cummings model except that the reservoir operators are fermionic, $\left\{b_{\omega}, b_{\omega^{\prime}}^{\dagger}\right\}=\delta\left(\omega-\omega^{\prime}\right) 1$. Also, we consider the reservoir at temperature $T$ and chemical potential $\mu$ coupled with an energy-independent spectral density $\Gamma=$ const. This is the most basic model of transient electron tunneling from a localized state. Even though it ignores interaction effects, its propagator is feature rich. This was noted in recent work [102], but the nontrivial relations between $\mathcal{K}$ and $\mathcal{G}$ and their spectra noted below were overlooked. The diagonal representation of $\Pi$ reads [Ref. [102], Eq. (E1)]

$$
\begin{aligned}
\Pi(t)= & \left.\sum_{\eta= \pm} e^{[i \eta \varepsilon-(1 / 2) \Gamma] t} \mid d_{\eta}^{\dagger}\right)\left(d_{\eta}^{\dagger} \mid\right. \\
& \left.\left.+\frac{1}{2}[\mid \mathbb{1})+\mathrm{p}(t) \mid(-\mathbb{1})^{N}\right)\right](\mathbb{1} \mid \\
& \left.+e^{-\Gamma t} \frac{1}{2} \mid(-\mathbb{1})^{N}\right)\left[\left((-\mathbb{1})^{N} \mid-\mathrm{p}(t)(\mathbb{1} \mid],\right.\right.
\end{aligned}
$$

where $\left.d_{+} \equiv d^{\dagger}, d_{-} \equiv d, \mid O\right) \equiv O$, and $\left(O \mid \equiv \operatorname{tr}\left(O^{\dagger} \bullet\right)\right.$ for an operator $O$. In contrast to the Jaynes-Cummings model, its eigenvectors depend on time through the function

$$
\begin{aligned}
\mathrm{p}(t)= & \sum_{\eta= \pm} \eta \operatorname{Im}\left[\frac{e^{-(\pi T+i \epsilon) t}}{\pi \sinh (\Gamma t / 2)} \Phi\left(e^{-2 \pi T t}, 1, \frac{1}{2}+\frac{i \epsilon+\eta \Gamma / 2}{2 \pi T}\right)\right. \\
& \left.+\frac{e^{\eta \Gamma t / 2}}{\pi \sinh (\Gamma t / 2)} \Psi\left(\frac{1}{2}+\frac{i \epsilon+\eta \Gamma / 2}{2 \pi T}\right)\right]
\end{aligned}
$$

involving Lerch $(\Phi)$ and digamma $(\Psi)$ functions with $\epsilon=\varepsilon-\mu$. This richer structure is also reflected by the analytic properties of the propagator (Ref. [102], Appendix D),

$$
\begin{aligned}
\hat{\Pi}(\omega)= & \left.\sum_{\eta= \pm} \frac{i}{\omega+\eta \varepsilon+i \frac{\Gamma}{2}} \mid d_{\eta}^{\dagger}\right)\left(d_{\eta}^{\dagger} \mid\right. \\
& \left.\left.+\frac{i}{\omega} \frac{1}{2}[\mid \mathbb{1})+\hat{\mathrm{k}}\left(\omega+i \frac{\Gamma}{2}\right) \mid(-\mathbb{1})^{N}\right)\right](\mathbb{1} \mid \\
& \left.+\frac{i}{\omega+i \Gamma} \frac{1}{2} \mid(-\mathbb{1})^{N}\right)\left[\left((-\mathbb{1})^{N} \mid-\hat{\mathrm{k}}\left(\omega+i \frac{\Gamma}{2}\right)(\mathbb{\mathbb { 1 }} \mid],\right.\right.
\end{aligned}
$$

expressed in the Laplace transform $\hat{\mathrm{k}}(\omega) \equiv \int_{0}^{\infty} d t e^{i \omega t} \mathrm{k}(t)$ of $\mathrm{k}(t) \equiv 2 T \sin [(\varepsilon-\mu) t] / \sinh [\pi T t]$. Its poles, listed in Table II, include two infinite series for $T>0$, which merge into branch cuts as $T \rightarrow 0$.

The generator $\mathcal{G}(t)=i \dot{\Pi}(t) \Pi^{-1}(t) \quad$ [Ref. [102], Eq. (B14)],

$$
\begin{aligned}
\mathcal{G}(t)= & \left.\sum_{\eta= \pm}\left(-\eta \varepsilon-i \frac{1}{2} \Gamma\right) \mid d_{\eta}^{\dagger}\right)\left(d_{\eta}^{\dagger} \mid\right. \\
& \left.-i \Gamma \frac{1}{2} \mid(-\mathbb{1})^{N}\right)\left[\left((-\mathbb{1})^{N} \mid-\mathrm{g}(t)(\mathbb{1} \mid],\right.\right.
\end{aligned}
$$

is obtained with $\mathrm{g}(t)=\int_{0}^{t} d s e^{-(1 / 2) \Gamma s} \mathrm{k}(s)$, which is related to $\mathrm{p}(t)=\Gamma /\left(1-e^{-\Gamma t}\right) \int_{0}^{t} d s e^{-\Gamma(t-s)} \mathrm{g}(s)$. The evolution changes its Markovian character from CP divisible $[|\mathrm{g}(t)| \leq 1]$ close to resonance to nondivisible sufficiently far from resonance. The kernel $\hat{\mathcal{K}}(\omega)=\omega \mathcal{I}-i \hat{\Pi}^{-1}(\omega)$ can be expressed as [Ref. [102], Eq. (D13)]

TABLE II. Resonant level model, $n=0,1,2, \ldots$.

\begin{tabular}{ll}
\hline \hline Poles $\hat{\Pi}(\omega)$ & Eigenvalues $\mathcal{G}(\infty)$ \\
\hline$\omega_{0}=0$ & $g_{0}=0$ \\
$\omega_{1}=+(\varepsilon-\mu)-i \frac{1}{2} \Gamma$ & $g_{1}=+(\varepsilon-\mu)-i \frac{1}{2} \Gamma$ \\
$\omega_{2}=-(\varepsilon-\mu)-i \frac{1}{2} \Gamma$ & $g_{2}=-(\varepsilon-\mu)-i \frac{1}{2} \Gamma$ \\
$\omega_{3}=-\Gamma$ & $g_{3}=-\Gamma$ \\
$\omega_{4+2 n}=\omega_{1}-i \pi T(2 n+1) \leftarrow$ Possibly closer to real axis \\
$\omega_{5+2 n}=\omega_{2}-i \pi T(2 n+1) \leftarrow$ than $\omega_{3} !$ \\
\hline \hline
\end{tabular}




$$
\begin{aligned}
\hat{\mathcal{K}}(\omega)= & \left.\sum_{\eta= \pm}\left(-\eta \varepsilon-i \frac{1}{2} \Gamma\right) \mid d_{\eta}^{\dagger}\right)\left(d_{\eta}^{\dagger} \mid\right. \\
& \left.-i \Gamma \frac{1}{2} \mid(-\mathbb{1})^{N}\right)\left[\left((-\mathbb{1})^{N} \mid-\hat{\mathrm{k}}\left(\omega+i \frac{1}{2} \Gamma\right)(\mathbb{\mathbb { 1 }} \mid] .\right.\right.
\end{aligned}
$$

Unlike the Jaynes-Cummings model, none of these superoperators commute with themselves at different time or frequency or parameter values (on which their eigenvectors depend) nor with each other [since $\mathrm{p}(t), \mathrm{k}(t), \mathrm{g}(t)$ all differ]. We now show that, nevertheless, the sampling relation (21) explicitly holds.

Table II shows that the four eigenvalues of $\mathcal{G}(\infty)$ indeed coincide with four of the poles of $\hat{\Pi}(\omega)$, which coincide with the four frequency-independent eigenvalues of $\hat{\mathcal{K}}(\omega)$. However, the propagator $\hat{\Pi}(\omega)$ has infinitely many more poles $\left\{\omega_{n}\right\}_{n \geq 4}$ which arise from the function $\hat{\mathrm{k}}\left(\omega+i \frac{1}{2} \Gamma\right)$ located in the eigenvectors of $\hat{\mathcal{K}}(\omega)$. These are not sampled as explained after Eq. (19). For $T \leq \Gamma /(2 \pi)$ some of these nonsampled poles lie in between the sampled poles $\omega_{1}, \omega_{2}$, and $\omega_{3}$ and form branch cuts as $T \rightarrow 0$.

In Table III we illustrate how $\mathcal{G}(\infty)$ also nontrivially samples the eigenvectors of $\hat{\mathcal{K}}(\omega)$ as follows. (i) We collect one right eigenvector from each of the four different superoperators $\hat{\mathcal{K}}(0), \quad \hat{\mathcal{K}}\left( \pm \varepsilon-i \frac{1}{2} \Gamma\right), \quad$ and $\hat{\mathcal{K}}(-i \Gamma)$. (ii) This gives four right vectors $\left.\left.\mid \hat{k}_{j_{i}}\left(g_{i}\right)\right)=\mid g_{i}\right)$. (iii) From this set one algebraically constructs a set of biorthonormal covectors $\left(g_{i}^{\prime} \mid\right.$. This way we remarkably obtain the left and right eigenvectors of $\mathcal{G}(\infty)$ as given by Eq. (51) using the analytic property $\mathrm{g}(\infty)=\hat{\mathrm{k}}\left(i \frac{1}{2} \Gamma\right)$. Note in particular that one would not obtain the correct left eigenvectors of $\mathcal{G}(\infty)$ by naively sampling the left pole eigenvectors of the kernels. For eigenvalue $g_{3}=-i \Gamma$ a difference arises as indicated by the two arrows in Table III.

We observe that for the resonant level model each eigenvalue pole is sampled precisely once by $\mathcal{G}(\infty)$. Combined with the mere assumption that $\mathcal{G}(\infty)$ is diagonalizable, the sampling relation (21) thus completely determines this superoperator, because it exhausts the number of eigenvalue poles $\left(d^{2}=4\right)$.
For the resonant level model the numerical stationary iteration (Sec. IVA) starting from any initial $\mathcal{G}^{(0)}(\infty)$ also converges to the exact stationary generator. This holds for all parameters of the model. Strikingly, using $\mathcal{G}^{(0)}(\infty)=$ $\hat{\mathcal{K}}(0)$ as a starting point the iteration terminates right away at the zeroth iteration, implying an exact relation [125]:

$$
\mathcal{G}(\infty)=\hat{\mathcal{K}}(0) .
$$

One verifies the relation (53) by comparing Eqs. (52) and (51) again using $\mathrm{g}(\infty)=\hat{\mathrm{k}}\left(i \frac{1}{2} \Gamma\right)$.

The transient iteration (Sec. IV B) starting from the constant ansatz $\mathcal{G}^{(0)}(t)=\hat{\mathcal{K}}(0)$ does not terminate immediately, because the evolution is not a semigroup. However, for this ansatz the first transient iteration does give the exact solution, $\mathcal{G}^{(1)}(t)=\int_{0}^{t} d s \mathcal{K}(t-s) e^{i \hat{\mathcal{K}}(0)(t-s)}=\mathcal{G}(t)$, again for all parameters of the model. This reflects an exact relation [Ref. [102], Eqs. (52a) and (D15)],

$$
\mathcal{G}(t)=\int_{0}^{t} d s \mathcal{K}(t-s),
$$

which for $t \rightarrow \infty$ again implies Eq. (53). In Fig. 5 we show the time dependence of the occupations for the zeroth and first iteration. Unlike the Jaynes-Cummings model, the level initially actually fills up more before decaying to the empty stationary state, an effect caused by time dependence of eigenvectors of $\Pi(t)$ [Eq. (48)]. This reentrant behavior is completely produced in one step by $\mathcal{G}^{(1)}(t)$ from the Markov semigroup approximation $\mathcal{G}^{(0)}=\hat{\mathcal{K}}(0)=\mathcal{G}(\infty)$, which can never capture an initial growth in the "wrong direction".

In fact, any trace-preserving constant ansatz $\mathcal{G}^{(0)}(t)=X$ gives the exact solution after one iteration, $\mathcal{G}^{(1)}(t)=\mathcal{G}(t)$, as shown in Appendix A. Furthermore, starting from an arbitrary time-constant superoperator, $\mathcal{G}^{(0)}=X \neq 0$, the second transient iteration always reaches the fixed point, because the first iteration $\mathcal{G}^{(1)}(t)$ produces a trace-preserving generator [Eq. (12)]. That two iterations suffice for all parameters of the model is remarkable since this includes the extended parameter regime where the level is sufficiently off resonant and the evolution is not $\mathrm{CP}$ divisible

TABLE III. Sampling of memory kernel $\hat{\mathcal{K}}(\omega)$ by the stationary generator $\mathcal{G}(\infty)$ [Eq. (21)]. Left-hand columns: for each different superoperator $\hat{\mathcal{K}}\left(g_{i}\right)$ we list one pole eigenvalue with its left and right eigenvector. Right-hand columns: collecting the right eigenvectors from $\hat{\mathcal{K}}\left(g_{i}\right)$ and biorthonormalizing, we construct the left eigenvectors $\left(g_{i}^{\prime} \mid\right.$. Row $i=1,2$ corresponds to $\eta= \pm$.

\begin{tabular}{lrrlrrl}
\hline \hline & (iv) Do not copy these & $\hat{\mathcal{K}}\left(g_{i}\right)$ & (i) Copy these & (iii) Biorthogonalize & $\mathcal{G}(\infty)$ & (ii) Collect here \\
\cline { 2 - 7 }$i$ & $\left(\hat{k}_{j_{i}}^{\prime}\left(g_{i}\right) \mid\right.$ & $\hat{k}_{j_{i}}\left(g_{i}\right)$ & $\left.\mid \hat{k}_{j_{i}}\left(g_{i}\right)\right)$ & $\left(g_{i}^{\prime} \mid\right.$ & $g_{i}$ & $\left.\mid g_{i}\right)$ \\
\hline 0 & $(\mathbb{1} \mid$ & 0 & $\left.\left.\frac{1}{2}[\mid \mathbb{1})+\hat{\mathrm{k}}\left(i \frac{\Gamma}{2}\right) \mid(-\mathbb{1})^{N}\right)\right]$ & $(\mathbb{1} \mid$ & 0 & $\left.\left.\frac{1}{2}[\mid \mathbb{1})+\hat{\mathrm{k}}\left(i \frac{\Gamma}{2}\right) \mid(-\mathbb{1})^{N}\right)\right]$ \\
1,2 & $\downarrow\left(d_{\eta}^{\dagger} \mid\right.$ & $-\eta \varepsilon-i \frac{1}{2} \Gamma$ & $\left.\mid d_{\eta}^{\dagger}\right)$ & $\downarrow\left(d_{\eta}^{\dagger} \mid\right.$ & $-\eta \varepsilon-i \frac{1}{2} \Gamma$ & $\left.\mid d_{\eta}^{\dagger}\right)$ \\
3 & $\frac{1}{2}\left[\left((-\mathbb{1})^{N} \mid-\hat{\mathrm{k}}\left(-i \frac{\Gamma}{2}\right)(\mathbb{1} \mid]\right.\right.$ & $-i \Gamma$ & $\left.\mid(-\mathbb{1})^{N}\right)$ & $\frac{1}{2}\left[\left((-\mathbb{1})^{N} \mid-\hat{\mathrm{k}}\left(i \frac{\Gamma}{2}\right)(\mathbb{1} \mid]\right.\right.$ & $-i \Gamma$ & $\left.\mid(-\mathbb{1})^{N}\right)$ \\
\hline \hline
\end{tabular}




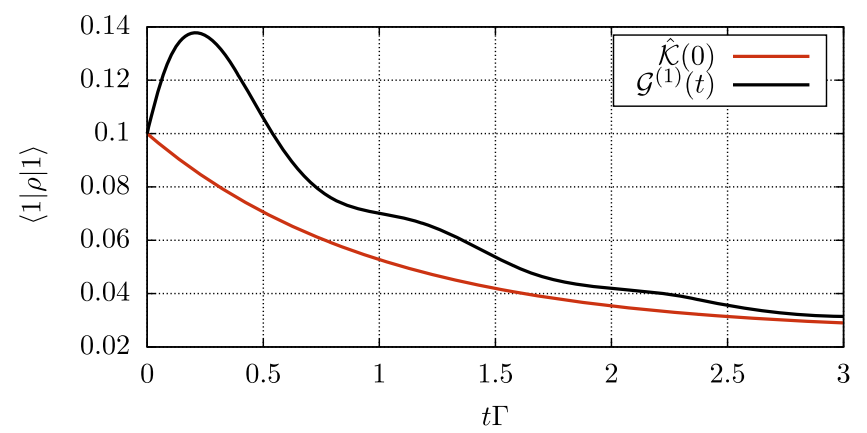

FIG. 5. Decay of the occupation $\langle 1|\rho(t)| 1\rangle$ in the resonant level model for $\varepsilon-\mu=2 \pi \Gamma, \quad T=0.1 \times \Gamma /(2 \pi)$ obtained from $\mathcal{G}^{(0)}(t)=\hat{\mathcal{K}}(0)$ and $\mathcal{G}^{(1)}(t)=\mathcal{G}(t)$. The former corresponds to a Markov semigroup approximation using $\hat{\mathcal{K}}(0)$, which is never able to describe the initial growth of the occupation away from the stationary value. When used as initial guess in the transient iteration the exact solution is recovered by the fixed-point equation (10) after a single step.

[94,102]. For comparison, in the underdamped regime where the Jaynes-Cummings model is not CP-divisible many iterations are required (Fig. 3). The termination of the fixed-point iteration is closely related to truncations of (renormalized) coupling expansions for $\mathcal{K}(t)$ and $\Pi(t)$ for fermionic models, which occur in the absence of interactions and for energy-independent coupling [7,8,102]. This indicates that the number of iterations is related to dynamically generated many-body effects.

\section{SUMMARY AND OUTLOOK}

We found the general connection between two canonical approaches to the dynamics of open quantum systems, the time-local and time-nonlocal quantum master equation. This relation extends the response function of an open system-the frequency-domain memory kernel $\hat{\mathcal{K}}(\omega)$ - to a functional mapping of superoperator functions of time of which the generator is a fixed point: $\mathcal{G}\left(t, t_{0}\right)=\hat{\mathcal{K}}[\mathcal{G}]\left(t, t_{0}\right)$. The fixed-point property expresses that the generator is a characteristic "frequency" of the evolution produced by the memory kernel. This is very similar to how pole frequencies characterize the response of linear systems in physical sciences and engineering [126]. In our general quantum setting, we showed how the fixed-point equation provides a self-consistent solution of the complicated time-domain gradient expansion. Interestingly, this also revealed a connection of the time-convolutionless approach to a Moyal formulation of quantum theory of open systems.

We obtained several general insights into the role of the frequency dependence of the memory kernel. We precisely determined how the stationary generator $\mathcal{G}(\infty)$ samples the right eigenvectors and eigenvalues of the memory kernel $\hat{\mathcal{K}}(\omega)$ at zero and nonzero characteristic frequencies of the evolution. The sampled frequencies form a finite subset of the exact poles of the frequency-domain evolution as obtained by the Laplace resolvent method in the timenonlocal approach. Remarkably, knowing only the location of these poles in the complex plane in principle suffices to completely construct the stationary generator $\mathcal{G}(\infty)$ from the memory kernel, significantly simplifying analytical calculations. This generator may also be obtained numerically by iterating the stationary fixed-point equation.

Similarly, the full transient generator may be obtained from the memory kernel by iterating the functional fixedpoint equation (10). At each iteration the approximate generator is both initially and asymptotically accurate. Importantly, this iteration strategy only works if the generator is a locally stable fixed point of the kernel functional and we have shown that even time-singular generators can be locally stable. We also showcased an evolution with time-dependent eigenvectors whose generator is exactly found after at most two iterations. Since our results apply quite generally and can be tailored to both numerical [22,23] and analytical [20,21,24] applications, they seem relevant to the challenging problems of strongly interacting open quantum systems dominated by nonperturbative dissipation and memory effects. Altogether, this provides new starting points for combining well-developed memory kernel formalisms to access the advantages of a time-local description. We conclude by outlining several such applications.

(Non-)Markovianity and microscopic models.-As mentioned in the Introduction, the most obvious application lies in the study of the divisibility properties of the dynamics, which are directly accessible via the time-local generator $\mathcal{G}$. From its time-dependent canonical form [42] one can extract both the jump rates (characterizing $\mathrm{CP}$ divisibility) and the jump operators (characterizing $\mathrm{P}$ divisibility) [53,54], encompassing different degrees of (non-)Markovianity [70]. However, the impressive progress in understanding of dynamics and information in general terms contrasts with the enduring limitation of their application to microscopic models. Our key result (10) provides a new path from accurate memory kernels calculated within the well-developed time-nonlocal formalism to the time-local description required for these problems.

Geometry, topology, and transport in open systems.Similarly, the study of geometric [63,64] and topological phases [66,67] hinges on a time-local description. Starting from the time-local QME, already in the leading adiabatic approximation to the dynamics,

$$
\begin{aligned}
\mid \rho(t)) & \left.=\mathcal{T}_{\leftarrow} e^{-i \int_{0}^{t} \mathcal{G}(t)} \mid \rho(0)\right) \\
& \left.\approx \sum_{i} \mid g_{i}(t)\right)\left(\bar{g}_{i}(t) \mid \rho(0)\right) e^{\int_{0}^{t} d s\left[-i g_{i}(s)-\left(\bar{g}_{i}(s) \mid \partial_{s} g_{i}(s)\right)\right],}
\end{aligned}
$$

one finds that the time-instantaneous eigenvalues and eigenvectors of $\mathcal{G}(t)$-not those of the memory kernel 
$\mathcal{K}(t)$ - determine the dynamic and geometric phase of the open system, respectively. For stationary driving one can expand these quantities around their stationary values with parametric time dependence. Our stationary fixedpoint equation (15) describes how these required quantities are nontrivially related to eigenvalues and eigenvectors of the more accessible memory kernel $\mathcal{K}$. As illustrated in Sec. III D, this procedure automatically includes the full memory expansion of the kernel [36].

These geometric phases of state evolution subsume [127] those of observables [128-131] and their full-counting statistics $[68,132]$ as a special case by incorporating an ideal counter into the state evolution [133,134]. This immediately implies that our fundamental relation (10) for state evolution translates to a nontrivial relation between the time-nonlocal kernel and time-local generator that govern the dynamics of the full-counting statistics, i.e., the transport equations. The full-counting statistics is pivotal in quantum thermodynamics [73,74], adiabatic operations [72], energy backflow [135], nonequilibrium fluctuation relations [69], and studies of information [136] and entropy production $[137,138]$.

Perturbation expansions of $\mathcal{G}(t)$. - Our fixed-point relation (10) also allows challenges faced by perturbative calculations of $\mathcal{G}[17,34,35,139]$ to be addressed. Given some expansion of the memory kernel, $\mathcal{K}=\mathcal{K}^{(1)}+\mathcal{K}^{(2)}+\cdots$, a corresponding series for $\mathcal{G}=\mathcal{G}^{(1)}+\mathcal{G}^{(2)}+\cdots$ is obtained in terms of the memory kernel alone without introducing any new formalism. This is done by expanding Eq. (10) and organizing both sides of the equation order by order. The first orders of $\mathcal{G}$ are then given by

$$
\begin{aligned}
\mathcal{G}^{(1)}\left(t, t_{0}\right)= & \int_{t_{0}}^{t} d s \mathcal{K}^{(1)}(t, s), \\
\mathcal{G}^{(2)}\left(t, t_{0}\right)= & \int_{t_{0}}^{t} d s \mathcal{K}^{(2)}(t, s) \\
& +i \int_{t_{0}}^{t} d s \int_{s}^{t} d \tau \mathcal{K}^{(1)}(t, s) \mathcal{G}^{(1)}\left(\tau, t_{0}\right) .
\end{aligned}
$$

This reveals a recursive structure of perturbative $\mathcal{G}$ expansions. Expanding in powers of the system-environment coupling one recovers the approach of Ref. [34]. However, Eq. (56) is flexible and can also be applied to expansions around a known dissipative solution, which has no Hamiltonian formulation. An interesting example is the $T=\infty$ solution of strongly interacting, wideband limit transport models [7,8,93], which leads to a powerful renormalized perturbation theory [8]. This may be a step up for a renormalization group [20,21,24,26,27] treatment for time-local generators.

Nonperturbative semigroups and initial slippage.Our results can be used to gain detailed insight into approximation strategies in the nonperturbative regime. In particular, the superoperator $\mathcal{S}$ in Eqs. (22)-(24) is crucial for the "slippage of the initial condition," a well-known procedure aiming to improve Markovian approximations [95-99]. In contrast to most previous works, we can derive definite statements about the nonperturbative quantity $\mathcal{S}$. This has been explored in Ref. [94] for the most favorable situation where a Markov approximation using the exact Markovian generator $\mathcal{G}(\infty)$ is improved upon using the exact slippage superoperator $\mathcal{S}$. Even in this case subtle failures can arise, which are totally unexpected within the time-local formalism used to set up the slippage correction. Already for the example of the resonant level a dramatic breakdown occurs around apparently "innocent" isolated physical parameter points, even though away from these points it gives a considerably improved nonsemigroup approximation. However, this behavior can be clearly understood [94] using the connection (21) to the time-nonlocal memory kernel and applies generally to strongly interacting fermionic transport models far from equilibrium.

Fixed-point analysis.-Aside from these increasingly technical applications, perhaps the most intriguing implication of our main result (10) is the possibility of analyzing the fixed-point iteration using ideas borrowed from renormalization group transformations in statistical physics [140], such as linearization around fixed points, scaling, etc. In the iteration (30) each new approximation $\mathcal{G}^{(n)}$ incorporates more of the memory integral over $\mathcal{K}$ in a stepwise fashion. The apparent local stability of the fixed point of this discrete flow in the functional superoperator space and its range of attraction must somehow be related to physical retardation properties of the open system. One could envisage comparing and perhaps even classifying opensystem dynamics based on the nature of these discrete flows.

\section{ACKNOWLEDGMENTS}

We thank Y.-T. Lin, J. Schulenborg, J. Splettstoesser, B. Vacchini, M. Pletyukhov, C. Timm, Y. Mokrousov, and F. Lux for useful discussions. K. N. and V. B. acknowledge support by the Deutsche Forschungsgemeinschaft (RTG 1995).

\section{APPENDIX A: ITERATION IN THE RESONANT LEVEL MODEL}

For the resonant level model both the stationary (Sec. IV A) and transient (Sec. IV B) fixed-point iteration terminate at the first step when starting from any zero-tracepreserving superoperator $X$, i.e., $\operatorname{Tr} X \bullet=0$. This can be seen by writing the time-nonlocal part of the kernel as $[8,102]$

$$
\mathcal{K}_{N}(t)=-i \Gamma \mathrm{k}(t) e^{-\Gamma t / 2} G_{+}^{+} G_{-}^{+},
$$

in terms of fermionic "creation" superoperators [7] satisfying $\left(G_{\eta}^{+}\right)^{2}=0$ and thus $G_{+}^{+} G_{-}^{+} G_{\eta}^{+}=0$. Using "second 
quantization" for superoperators $[8,102]$ one expands any zero-trace-preserving superoperator $X$ in terms of products of superfields and verifies that in each term $G_{\eta}^{+}$stands on the far left. This implies

$$
\mathcal{K}_{N}(t-s) e^{i X(t-s)}=\mathcal{K}_{N}(t-s),
$$

and by Eq. (30) the transient [Eq. (54)] and stationary [Eq. (53)] iteration find the exact generator in a single step. Starting from an arbitrary superoperator $X$, the first iteration will make it zero trace by Eq. (12), and by the above result the second iteration will be converged.

\section{APPENDIX B: EXACT SUMMATION OF THE MEMORY EXPANSION OF THE TIME- NONLOCAL QME}

In Sec. III D of the text we mentioned that the memory expansions of Refs. [37,38] are contained in our fixed-point relation (10). Here we give an explicit formula for all terms. Moreover, we sum the series to a self-consistent form and recover our key results (10) and (15).

Memory expansion.-We essentially follow the approach of Ref. [37] noting that we have verified that Ref. [38] achieves exactly the same thing by manipulating partial integrations. Both works start from the timenonlocal QME (7) and construct the time-local QME (5). Importantly, no weak coupling approximation is made in these works, but they do restrict attention to the stationary limit $t_{0} \rightarrow-\infty$ by constructing the approximate time-local QME $(d / d t) \Pi\left(t-t_{0}\right) \approx-i \mathcal{G}(\infty) \Pi\left(t-t_{0}\right)$. This is the nonperturbative Markovian semigroup approximation discussed in Sec. III C. Reference [37] considers only the leading memory correction (27). Here we make none of the mentioned assumptions and specialize to the case of Refs. [37,38] only at the end [Eq. (B9)].

Thus, the summation of the memory expansion amounts to the construction of $\mathcal{G}\left(t, t_{0}\right)$ from $\mathcal{K}(t, s)$ such that we have $(d / d t) \Pi\left(t, t_{0}\right)=-i \int_{t_{0}}^{t} d s \mathcal{K}(t, s) \Pi\left(s, t_{0}\right)=$ $-i \mathcal{G}\left(t, t_{0}\right) \Pi\left(t, t_{0}\right)$. In the main text this was solved by exploiting the divisor, $\mathcal{G}\left(t, t_{0}\right)=\int_{t_{0}}^{t} d s \mathcal{K}(t, s) \Pi\left(s, t \mid t_{0}\right)$. In our formulation, the approach taken in Refs. [37,38] amounts to computing the divisor as

$$
\begin{aligned}
\Pi\left(s, t \mid t_{0}\right) & =\Pi\left(s, t_{0}\right) \Pi\left(t, t_{0}\right)^{-1} \\
& =\sum_{k=0}^{\infty} \frac{1}{k !}(-1)^{k}(t-s)^{k} \mathcal{F}^{k}\left(t, t_{0}\right)
\end{aligned}
$$

by inserting the memory expansion $\Pi\left(s, t_{0}\right)=$ $\sum_{k}(1 / k !)(-1)^{k}(t-s)^{k} \partial_{t}^{k} \Pi\left(t, t_{0}\right)$ of quantities in the past time $s$ around the present time $t>s$. For example, Eq. (27) discussed in Ref. [37] corresponds to the $k=0,1$ terms. Here the superoperator-valued Taylor coefficients $\mathcal{F}^{k}\left(t, t_{0}\right)$ are the time-local generators of the $k$ th derivative of the propagator:

$$
\partial_{t}^{k} \Pi\left(t, t_{0}\right)=\mathcal{F}^{k}\left(t, t_{0}\right) \Pi\left(t, t_{0}\right) .
$$

Written as $\mathcal{F}^{k}\left(t, t_{0}\right):=\left[\partial_{t}^{k} \Pi\left(t, t_{0}\right)\right] \Pi\left(t, t_{0}\right)^{-1}$, they are easily shown to obey the recursion relation,

$\mathcal{F}^{k}\left(t, t_{0}\right)=\partial_{t} \mathcal{F}^{k-1}\left(t, t_{0}\right)+\mathcal{F}^{k-1}\left(t, t_{0}\right)\left[-i \mathcal{G}\left(t, t_{0}\right)\right]$,

with starting condition $\mathcal{F}^{0}\left(t, t_{0}\right)=\mathcal{I}$ giving, for instance,

$$
\begin{aligned}
\mathcal{F}^{1}\left(t, t_{0}\right)= & -i \mathcal{G}\left(t, t_{0}\right), \\
\mathcal{F}^{2}\left(t, t_{0}\right)= & -i \partial_{t} \mathcal{G}\left(t, t_{0}\right)+\left[-i \mathcal{G}\left(t, t_{0}\right)\right]^{2}, \\
\mathcal{F}^{3}\left(t, t_{0}\right)= & -i \partial_{t}^{2} \mathcal{G}\left(t, t_{0}\right)+\left[-i \mathcal{G}\left(t, t_{0}\right)\right]\left[-i \partial_{t} \mathcal{G}\left(t, t_{0}\right)\right] \\
& +2\left[-i \partial_{t} \mathcal{G}\left(t, t_{0}\right)\right]\left[-i \mathcal{G}\left(t, t_{0}\right)\right]+\left[-i \mathcal{G}\left(t, t_{0}\right)\right]^{3} .
\end{aligned}
$$

This suggests inserting the ansatz

$$
\begin{aligned}
\mathcal{F}^{k}\left(t, t_{0}\right)= & \sum_{n=1}^{k} \sum_{p_{1}=0}^{k-n} \ldots \sum_{p_{n}=0}^{k-n} \delta_{k-n, p_{1}+\cdots+p_{n}} \\
& \times F_{p_{1} \ldots p_{n}}^{n}\left[-i \partial_{t}^{p_{1}} \mathcal{G}\left(t, t_{0}\right)\right] \ldots\left[-i \partial_{t}^{p_{n}} \mathcal{G}\left(t, t_{0}\right)\right]
\end{aligned}
$$

and deriving the recursion relations for the coefficients,

$$
\begin{gathered}
F_{p_{1} \ldots p_{n}}^{n}=\sum_{j=1}^{n} F_{p_{1} \ldots\left(p_{j}-1\right) \ldots p_{n}}^{n}, \quad \text { for } p_{n} \geq 1 \\
F_{p_{1} \ldots p_{n-1} 0}^{n}=\sum_{j=1}^{n-1} F_{p_{1} \ldots\left(p_{j}-1\right) \ldots p_{n-1} 0}^{n}+F_{p_{1} \ldots p_{n-1}}^{n-1} .
\end{gathered}
$$

Together with the starting conditions $F_{0}^{1}=1$, these define all the coefficients of the memory expansion. Construction of the general solution of the recursion equations (B6) is very cumbersome and hides the elegant functional fixed-point relation.

Fixed-point equation.-We now show that the result (B1b) with (B5) equivalently follows from our fixed-point relation by (10) inserting into Eq. (9) the memory expansion $\mathcal{G}\left(s_{i}, t_{0}\right)=\sum_{p_{i}}\left(1 / p_{i} !\right)\left(-t_{i}\right)^{p_{i}} \partial_{t}^{p_{i}} \mathcal{G}\left(t, t_{0}\right)$ and performing the nested integrations over variables $t_{i}=t-s_{i}$ : 
$\Pi\left(s, t \mid t_{0}\right)=\mathcal{T}_{\rightarrow} e^{-\int_{s}^{t} d \tau[-i \mathcal{G}]\left(\tau, t_{0}\right)}=\sum_{n=0}^{\infty}(-1)^{n} \int_{t>s_{n}>\cdots>s_{1}>s} d s_{n} \ldots d s_{1}\left[-i \mathcal{G}\left(s_{1}, t_{0}\right)\right] \ldots\left[-i \mathcal{G}\left(s_{n}, t_{0}\right)\right]=\sum_{k=0}^{\infty} \frac{(-1)^{k}}{k !}(t-s)^{k} \mathcal{F}^{k}\left(t, t_{0}\right)$

We obtain the explicit general form of all coefficients:

$$
\begin{aligned}
F_{p_{1} \ldots p_{n}}^{n} & =\frac{\left(n+\sum_{i} p_{i}\right) !}{\prod_{i} p_{i} ! \prod_{i=0}^{n-1}\left[\sum_{j=0}^{i} p_{n-j}\right]} \\
& =\prod_{i=1}^{n-1}\left(\begin{array}{c}
p_{n-i}+\sum_{j=1}^{i-1}\left(p_{n-j}+1\right) \\
p_{n-i}
\end{array}\right) \\
& =\prod_{i=1}^{n-1} F_{p_{i-1}, p_{i}+\cdots+p_{n}+(n-i)}^{2} .
\end{aligned}
$$

The factorization (B8b) into binomials shows that all coefficients are in fact integers. Using the form (B8c) one verifies [141] that the coefficients are indeed the solutions to the recursion relations (B6). With $\mathcal{G}\left(t, t_{0}\right)=$ $\int_{t_{0}}^{t} d s \mathcal{K}(t, s) \Pi\left(s, t \mid t_{0}\right)$ this establishes that the laborious determination of the coefficients and subsequent summation of the memory expansion (B1b) envisaged in Refs. [37,38] ultimately leads to our general functional fixed-point equation (10). Our derivation of this self-consistent equation in the main text circumvents all above complications by immediately identifying the divisor in Eq. (8). However, even if one is interested in generating memory expansions, our approach (B7) via the divisor is far simpler.

Noting the special coefficient values $F_{0 \ldots 0}^{n}=1$ we see that $\mathcal{F}^{k}=[-i \mathcal{G}]^{k}+$ (terms involving at least one time derivative of $\mathcal{G}$ ). Thus in the stationary limit where $\lim _{t_{0} \rightarrow-\infty} \partial_{t}^{k} \mathcal{G}\left(t, t_{0}\right)=0$ and $\lim _{t_{0} \rightarrow-\infty} \mathcal{G}\left(t, t_{0}\right)=\mathcal{G}(\infty)$,

$$
\begin{aligned}
\Pi(s-t \mid-\infty) & =\sum_{k=0}^{\infty} \frac{(-1)^{k}}{k !}(t-s)^{k}[-i \mathcal{G}(\infty)]^{k} \\
& =e^{i \mathcal{G}(\infty)(t-s)} .
\end{aligned}
$$

Inserted into $\mathcal{G}(\infty)=\int_{0}^{\infty} d s \mathcal{K}(t-s) \Pi(s-t \mid-\infty)$, we thus also directly recover our stationary fixed-point equation (15) for time-translational systems, $\mathcal{K}(t, s)=\mathcal{K}(t-s)$, by explicit summation of the stationary memory expansion. This is the specific expansion studied in Refs. [37,38].

\section{APPENDIX C: RELATION OF TIME-LOCAL GENERATOR AND GRADIENT OR MOYAL EXPANSION OF TIME-NONLOCAL QME}

The memory expansion (B1b) implies that the generator of the time-local QME $(d / d t) \Pi\left(t, t_{0}\right)=-i \mathcal{G}\left(t, t_{0}\right) \Pi\left(t, t_{0}\right)$ may be written as a gradient expansion,

$$
\begin{aligned}
\mathcal{G}\left(t, t_{0}\right) & =\sum_{k=0}^{\infty} \frac{(-1)^{k}}{k !}\left[\int_{t_{0}}^{t} d s \mathcal{K}(t, s)(t-s)^{k}\right] \mathcal{F}^{k}\left(t, t_{0}\right) \\
& =\left.\sum_{k=0}^{\infty} \frac{1}{k !}\left[\frac{\partial^{k}}{(-i \partial \omega)^{k}} \hat{\mathcal{K}}\left(\omega, t, t_{0}\right)\right]\right|_{\omega=0} \mathcal{F}^{k}\left(t, t_{0}\right),
\end{aligned}
$$

with frequency derivatives of the Laplace-like integral transform $\hat{\mathcal{K}}\left(\omega, t, t_{0}\right):=\int_{0}^{t-t_{0}} d s e^{i \omega s} \mathcal{K}(t, t-s)$ of the memory kernel ("finite-time Laplace transform"). Since $\mathcal{F}^{k}\left(t, t_{0}\right):=$ $\left[\partial_{t}^{k} \Pi\left(t, t_{0}\right)\right] \Pi\left(t, t_{0}\right)^{-1}=f\left(\mathcal{G}, \ldots, \partial_{t}^{k-1} \mathcal{G}\right)$ has no simple structure as function of $k$ [Eq. (B5)], it is not clear how the series can be summed, not even formally. This reflects that it arises from the nontrivial anti-time-ordered exponential (B7). If one instead considers its action on $\Pi\left(t, t_{0}\right)$,

$$
\begin{aligned}
\frac{d}{d t} \Pi\left(t, t_{0}\right) & =-i \sum_{k=0}^{\infty} \frac{(-1)^{k}}{k !}\left[\int_{t_{0}}^{t} d s \mathcal{K}(t, s)(t-s)^{k}\right] \partial_{t}^{k} \Pi\left(t, t_{0}\right) \\
& =-\left.i \sum_{k=0}^{\infty} \frac{1}{k !}\left[\frac{\partial^{k}}{(-i \partial \omega)^{k}} \hat{\mathcal{K}}\left(\omega, t, t_{0}\right)\right]\right|_{\omega=0} \partial_{t}^{k} \Pi\left(t, t_{0}\right),
\end{aligned}
$$

then the series can be formally summed to give a nonlinear time-frequency-domain differential operator. Its action on superoperator functions of $t$ must coincide with the linear action of $\mathcal{G}\left(t, t_{0}\right)$ on the superoperator evaluated at $t$ :

$\left.\hat{\mathcal{K}}\left(\omega, t, t_{0}\right) e^{i(\bar{\partial} / \partial \omega)(\vec{\partial} / \partial t)}\right|_{\omega=0} \Pi\left(t, t_{0}\right)=\mathcal{G}\left(t, t_{0}\right) \Pi\left(t, t_{0}\right)$.

Thus, $\mathcal{G}\left(t, t_{0}\right)$ here plays the role of a (superoperator-valued) eigenvalue of this time-domain differential operator. This differential operator is constructed as frequency-domain differential operator acting to the left on the memory kernel transform $\hat{\mathcal{K}}\left(\omega, t, t_{0}\right)$. The above follows the well-known Moyal approach [76,77] to quantum physics of closed systems, where one enforces locality at the price of introducing position- and momentum-space differential operators acting both to the right and to the left. Its extension to the time-nonlocal evolution of open systems within the densityoperator approach is thus closely related to the time-convolutionless approach based on the time-local equation (2).

Clearly, this formal relation between the generator and the memory kernel is easily written down. However, our functional fixed-point result (10) goes beyond this by explicitly expressing the action of the time-domain differential operator on the left-hand side of Eq. (C3), evaluating $\partial_{t}^{k} \Pi\left(t, t_{0}\right)=\mathcal{F}^{k}\left(t, t_{0}\right) \Pi\left(t, t_{0}\right)$ [Eq. (B5)], and summing the 
series to an anti-time-ordered exponential in terms of $\mathcal{G}\left(t, t_{0}\right)$. This is demonstrated by Eq. (B7) read in reverse order. As the main text shows, this makes the fixed-point relation a powerful analytical and numerical tool.

For time-translational systems, $\mathcal{K}(t, s)=\mathcal{K}(t-s)$, taking the stationary limit leads to the simplification $\lim _{t_{0} \rightarrow-\infty} \mathcal{F}^{k}\left(t, t_{0}\right)=[-i \mathcal{G}(\infty)]^{k}$, giving

$$
\begin{aligned}
\mathcal{G}(\infty) & =\sum_{k=0}^{\infty} \frac{(-1)^{k}}{k !}\left[\int_{-\infty}^{t} d s \mathcal{K}(t-s)(t-s)^{k}\right][-i \mathcal{G}(\infty)]^{k} \\
& =\left.\sum_{k=0}^{\infty} \frac{1}{k !}\left[\frac{\partial^{k}}{\partial \omega^{k}} \hat{\mathcal{K}}(\omega)\right]\right|_{\omega=0} \mathcal{G}(\infty)^{k},
\end{aligned}
$$

with frequency derivatives of the Laplace-transformed memory kernel $\hat{\mathcal{K}}(\omega)=\int_{0}^{\infty} d s \mathcal{K}(s) e^{i s \omega}$. In this case, the gradient expansion can be summed to give an alternative expression for our stationary fixed-point relation (15):

$$
\left.\hat{\mathcal{K}}(\omega) e^{(\bar{\partial} / \partial \omega) \mathcal{G}(\infty)}\right|_{\omega=0}=\mathcal{G}(\infty) .
$$

This gives a nonlinear differential operator acting to the left on superoperator functions of $\omega$ and is a mere formal expression of our stationary fixed-point equation $(15), \hat{\mathcal{K}}(\mathcal{G}(\infty)):=$ $\int_{-\infty}^{t} d s \mathcal{K}(t-s) e^{i \mathcal{G}(\infty)(t-s)}$. Equation (C5) extends the shift property for ordinary Laplace transforms, $e^{(\partial / \partial \omega) \Delta} \hat{f}(\omega)=$ $\hat{f}(\omega+\Delta)$, to our result (16) with superoperator-valued frequency argument $\Delta=\mathcal{G}(\infty)$. To ensure that the memory kernel generates a trace-preserving evolution [Eq. (12)], the frequency derivatives must stand on the right and therefore need to act to the left to accomplish the shift.

[1] S. Nakajima, On Quantum Theory of Transport Phenomena, Prog. Theor. Phys. 20, 948 (1958).

[2] R. Zwanzig, Ensemble Method in the Theory of Irreversibility, J. Chem. Phys. 33, 1338 (1960).

[3] M. Tokuyama and H. Mori, Statistical-Mechanical Approach to Random Frequency Modulations and the Gaussian Memory Function, Prog. Theor. Phys. 54, 918 (1975).

[4] M. Tokuyama and H. Mori, Statistical-Mechanical Theory of Random Frequency Modulations and Generalized Brownian Motions, Prog. Theor. Phys. 55, 411 (1976).

[5] We use the normalization $\int_{0}^{\infty} d s \bar{\delta}(s)=1$ for initial-value problems.

[6] H.-P. Breuer and F. Petruccione, The Theory of Open Quantum Systems (Oxford University Press, London, England, 2002).

[7] R. B. Saptsov and M. R. Wegewijs, Fermionic Superoperators for Zero-Temperature Non-Linear Transport: Real-Time Perturbation Theory and Renormalization Group for Anderson Quantum Dots, Phys. Rev. B 86, 235432 (2012).

[8] R. B. Saptsov and M. R. Wegewijs, Time-Dependent Quantum Transport: Causal Superfermions, Exact Fermion-Parity Protected Decay Modes, and Pauli Exclusion
Principle for Mixed Quantum States, Phys. Rev. B 90, 045407 (2014).

[9] E. B. Davies, Quantum Theory of Open Systems (Academic Press, London, 1976).

[10] S. A. Gurvitz and Y. S. Prager, Microscopic Derivation of Rate Equations for Quantum Transport, Phys. Rev. B 53, 15932 (1996).

[11] A. Oguri and R. Sakano, Exact Interacting Green's Function for the Anderson Impurity at High Bias Voltages, Phys. Rev. B 88, 155424 (2013).

[12] V. Gorini, A. Kossakowski, and E. C. G. Sudarshan, Completely Positive Dynamical Semigroups of $N$-Level Systems, J. Math. Phys. (N.Y.) 17, 821 (1976).

[13] G. Lindblad, On the Generators of Quantum Dynamical Semigroups, Commun. Math. Phys. 48, 119 (1976).

[14] J. T. Barreiro, M. Müller, P. Schindler, D. Nigg, T. Monz, M. Chwalla, M. Hennrich, C. F. Roos, P. Zoller, and R. Blatt, An Open-System Quantum Simulator with Trapped Ions, Nature (London) 470, 486 (2011).

[15] R. Blatt and C.F. Roos, Quantum Simulations with Trapped Ions, Nat. Phys. 8, 277 (2012).

[16] C. Gross and I. Bloch, Quantum Simulations with Ultracold Atoms in Optical Lattices, Science 357, 995 (2017).

[17] B. Vacchini and H.-P. Breuer, Exact Master Equations for the Non-Markovian Decay of a Qubit, Phys. Rev. A 81, 042103 (2010).

[18] A. Smirne and B. Vacchini, Nakajima-Zwanzig versus Time-Convolutionless Master Equation for the Non-Markovian Dynamics of a Two-Level System, Phys. Rev. A 82, 022110 (2010).

[19] N. Megier, D. Chruściński, J. Piilo, and W. T. Strunz, Eternal Non-Markovianity: From Random Unitary to Markov Chain Realisations, Sci. Rep. 7, 6379 (2017).

[20] H. Schoeller, A Perturbative Nonequilibrium Renormalization Group Method for Dissipative Quantum Mechanics, Eur. Phys. J. Special Topics 168, 179 (2009).

[21] H. Schoeller, Dynamics of Open Quantum Systems, arXiv:1802.10014.

[22] G. Cohen and E. Rabani, Memory Effects in Nonequilibrium Quantum Impurity Models, Phys. Rev. B 84, 075150 (2011).

[23] L. Kidon, H. Wang, M. Thoss, and E. Rabani, On the Memory Kernel and the Reduced System Propagator, J. Chem. Phys. 149, 104105 (2018).

[24] M. Pletyukhov and H. Schoeller, The Nonequilibrium Kondo Model: Crossover from Weak to Strong Coupling, Phys. Rev. Lett. 108, 260601 (2012).

[25] E. Y. Wilner, H. Wang, G. Cohen, M. Thoss, and E. Rabani, Bistability in a Nonequilibrium Quantum System with Electron-Phonon Interactions, Phys. Rev. B 88, 045137 (2013).

[26] C. J. Lindner and H. Schoeller, Dissipative Quantum Mechanics beyond the Bloch-Redfield Approximation: A Consistent Weak-Coupling Expansion of the Ohmic Spin Boson Model at Arbitrary Bias, Phys. Rev. B 98, 115425 (2018).

[27] C. J. Lindner, F. B. Kugler, V. Meden, and H. Schoeller, Renormalization Group Transport Theory for Open Quantum Systems: Charge Fluctuations in Multilevel Quantum 
Dots in and out of Equilibrium, Phys. Rev. B 99, 205142 (2019).

[28] A. Braggio, J. König, and R. Fazio, Full Counting Statistics in Strongly Interacting Systems: Non-Markovian Effects, Phys. Rev. Lett. 96, 026805 (2006).

[29] C. Flindt, T. Novotný, A. Braggio, M. Sassetti, and A.-P. Jauho, Counting Statistics of Non-Markovian Quantum Stochastic Processes, Phys. Rev. Lett. 100, 150601 (2008).

[30] K. H. Thomas and C. Flindt, Electron Waiting Times in Non-Markovian Quantum Transport, Phys. Rev. B 87, 121405(R) (2013).

[31] F. Shibata, Y. Takahashi, and N. Hashitsume, A Generalized Stochastic Liouville Equation. Non-Markovian versus Memoryless Master Equations, J. Stat. Phys. 17, 171 (1977).

[32] F. Shibata and T. Arimitsu, Expansion Formulas in Nonequilibrium Statistical Mechanics, J. Phys. Soc. Jpn. 49, 891 (1980).

[33] S. Chaturvedi and F. Shibata, Time-Convolutionless Projection Operator Formalism for Elimination of Fast Variables. Applications to Brownian Motion, Z. Phys. B 35, 297 (1979).

[34] H.-P. Breuer, B. Kappler, and F. Petruccione, The TimeConvolutionless Projection Operator Technique in the Quantum Theory of Dissipation and Decoherence, Ann. Phys. (N.Y.) 291, 36 (2001).

[35] C. Timm, Time-Convolutionless Master Equation for Quantum Dots: Perturbative Expansion to Arbitrary Order, Phys. Rev. B 83, 115416 (2011).

[36] J. Splettstoesser, M. Governale, J. König, and R. Fazio, Adiabatic Pumping through a Quantum Dot with Coulomb Interactions: A Perturbation Expansion in the Tunnel Coupling, Phys. Rev. B 74, 085305 (2006).

[37] L. D. Contreras-Pulido, J. Splettstoesser, M. Governale, J. König, and M. Büttiker, Time Scales in the Dynamics of an Interacting Quantum Dot, Phys. Rev. B 85, 075301 (2012).

[38] C. Karlewski and M. Marthaler, Time-Local Master Equation Connecting the Born and Markov Approximations, Phys. Rev. B 90, 104302 (2014).

[39] Á. Rivas, S. F. Huelga, and M. B. Plenio, Entanglement and Non-Markovianity of Quantum Evolutions, Phys. Rev. Lett. 105, 050403 (2010).

[40] D. Chruściński and A. Kossakowski, Markovianity Criteria for Quantum Evolution, J. Phys. B 45, 154002 (2012).

[41] A. Rivas, S. F. Huelga, and M. B. Plenio, Quantum NonMarkovianity: Characterization, Quantification and Detection, Rep. Prog. Phys. 77, 094001 (2014).

[42] M. J. W. Hall, J. D. Cresser, L. Li, and E. Andersson, Canonical Form of Master Equations and Characterization of Non-Markovianity, Phys. Rev. A 89, 042120 (2014).

[43] G. Amato, H.-P. Breuer, and B. Vacchini, Microscopic Modeling of General Time-Dependent Quantum Markov Processes, Phys. Rev. A 99, 030102(R) (2019).

[44] V. Reimer and M. R. Wegewijs, Density-Operator Evolution: Complete Positivity and the Keldysh Real-Time Expansion, SciPost Phys. 7, 12 (2019).
[45] D. Chruściński and A. Kossakowski, Sufficient Conditions for Memory Kernel Master Equation, Phys. Rev. A 94, 020103(R) (2016).

[46] D. Chruściński and A. Kossakowski, Generalized SemiMarkov Quantum Evolution, Phys. Rev. A 95, 042131 (2017).

[47] B. Vacchini, A. Smirne, E.-M. Laine, J. Piilo, and H.-P. Breuer, Markovianity and Non-Markovianity in Quantum and Classical Systems, New J. Phys. 13, 093004 (2011).

[48] B. Vacchini, Non-Markovian Master Equations from Piecewise Dynamics, Phys. Rev. A 87, 030101(R) (2013).

[49] F. Ciccarello, G. M. Palma, and V. Giovannetti, CollisionModel-Based Approach to Non-Markovian Quantum Dynamics, Phys. Rev. A 87, 040103(R) (2013).

[50] F. A. Wudarski, P. Należyty, G. Sarbicki, and D. Chruściński, Admissible Memory Kernels for Random Unitary Qubit Evolution, Phys. Rev. A 91, 042105 (2015).

[51] K. Siudzińska and D. Chruściński, Memory Kernel Approach to Generalized Pauli Channels: Markovian, SemiMarkov, and Beyond, Phys. Rev. A 96, 022129 (2017).

[52] D. Chruściński, A. Kossakowski, and A. Rivas, Measures of Non-Markovianity: Divisibility versus Backflow of Information, Phys. Rev. A 83, 052128 (2011).

[53] S. Wißmann, H.-P. Breuer, and B. Vacchini, Generalized Trace-Distance Measure Connecting Quantum and Classical Non-Markovianity, Phys. Rev. A 92, 042108 (2015).

[54] J. Bae and D. Chruściński, Operational Characterization of Divisibility of Dynamical Maps, Phys. Rev. Lett. 117, 050403 (2016).

[55] S. N. Filippov and D. Chruściński, Time Deformations of Master Equations, Phys. Rev. A 98, 022123 (2018).

[56] L. Li, M. J. W. Hall, and H. M. Wiseman, Concepts of Quantum Non-Markovianity: A Hierarchy, Phys. Rep. 759, 1 (2018).

[57] M. M. Wolf and J. I. Cirac, Dividing Quantum Channels, Commun. Math. Phys. 279, 147 (2008).

[58] A. Müller-Hermes, D. Reeb, and M. M. Wolf, Quantum Subdivision Capacities and Continuous-Time Quantum Coding, IEEE Trans. Inf. Theory 61, 565 (2015).

[59] E. Nielsen, J. K. Gamble, K. Rudinger, T. Scholten, K. Young, and R. Blume-Kohout, Gate Set Tomography, arXiv:2009.07301.

[60] R. Vasile, S. Olivares, M. G. A. Paris, and S. Maniscalco, Continuous-Variable Quantum Key Distribution in NonMarkovian Channels, Phys. Rev. A 83, 042321 (2011).

[61] E.-M. Laine, H.-P. Breuer, and J. Piilo, Nonlocal Memory Effects Allow Perfect Teleportation with Mixed States, Sci. Rep. 4, 4620 (2014).

[62] B. Bylicka, M. Tukiainen, D. Chruściński, J. Piilo, and S. Maniscalco, Thermodynamic Power of Non-Markovianity, Sci. Rep. 6, 27989 (2016).

[63] M. S. Sarandy and D. A. Lidar, Adiabatic Approximation in Open Quantum Systems, Phys. Rev. A 71, 012331 (2005).

[64] M. S. Sarandy and D. A. Lidar, Abelian and Non-Abelian Geometric Phases in Adiabatic Open Quantum Systems, Phys. Rev. A 73, 062101 (2006).

[65] D. O. Krimer and M. Pletyukhov, Few-Mode Geometric Description of a Driven-Dissipative Phase Transition in 
an Open Quantum System, Phys. Rev. Lett. 123, 110604 (2019).

[66] F. Li, J. Ren, and N. A. Sinitsyn, Quantum Zeno Effect as a Topological Phase Transition in Full Counting Statistics and Spin Noise Spectroscopy, Eur. Phys. Lett. 105, 27001 (2014).

[67] R.-P. Riwar, Fractional Charges in Conventional Sequential Electron Tunneling, Phys. Rev. B 100, 245416 (2019).

[68] N. A. Sinitsyn, The Stochastic Pump Effect and Geometric Phases in Dissipative and Stochastic Systems, J. Phys. A 42, 193001 (2009).

[69] R.-P. Riwar and J. Splettstoesser, Transport Fluctuation Relations in Interacting Quantum Pumps, arXiv:2010 .10482 .

[70] D. Chruściński and F. A. Wudarski, Non-Markovianity Degree for Random Unitary Evolution, Phys. Rev. A 91, 012104 (2015).

[71] M. Popovic, B. Vacchini, and S. Campbell, Entropy Production and Correlations in a Controlled NonMarkovian Setting, Phys. Rev. A 98, 012130 (2018).

[72] C. Uchiyama, Nonadiabatic Effect on the Quantum Heat Flux Control, Phys. Rev. E 89, 052108 (2014).

[73] J. Goold, M. Huber, A. Riera, L. del Rio, and P. Skrzypczyk, The Role of Quantum Information in Thermodynamics-A Topical Review, J. Phys. A 49, 143001 (2016).

[74] S. Vinjanampathy and J. Anders, Quantum Thermodynamics, Contemp. Phys. 57, 545 (2016).

[75] N. Megier, A. Smirne, and B. Vacchini, The Interplay between Local and Non-Local Master Equations: Exact and Approximated Dynamics, New J. Phys. 22, 083011 (2020).

[76] J. E. Moyal, Quantum Mechanics as a Statistical Theory, Math. Proc. Cambridge Philos. Soc. 45, 99 (1949).

[77] H. J. Groenewold, On the Principles of Elementary Quantum Mechanics (Springer, Dordrecht, 1946).

[78] J. Rammer and H. Smith, Quantum Field-Theoretical Methods in Transport Theory of Metals, Rev. Mod. Phys. 58, 323 (1986).

[79] S. Onoda, N. Sugimoto, and N. Nagaosa, Theory of NonEquilibirum States Driven by Constant Electromagnetic Fields-Non-Commutative Quantum Mechanics in the Keldysh Formalism, Prog. Theor. Phys. 116, 61 (2006).

[80] D. Sternheimer, Deformation Quantization: Twenty Years After, AIP Conf. Proc. 453, 107 (1998).

[81] C. Zachos, A Survey of Star Product Geometry, arXiv:hepth/0008010.

[82] N. Dittmann, J. Splettstoesser, and N. Helbig, Non-Adiabatic Dynamics in Single-Electron Tunneling Devices with Time-Dependent Density Functional Theory, Phys. Rev. Lett. 120, 157701 (2018).

[83] N. Dittmann, N. Helbig, and D. M. Kennes, Dynamics of the Anderson Impurity Model: Benchmarking a Nonadiabatic Exchange-Correlation Potential in Time-Dependent Density-Functional Theory, Phys. Rev. B 99, 075417 (2019).

[84] M. Hell, M. R. Wegewijs, and D. P. DiVincenzo, Coherent Backaction of Quantum Dot Detectors: Qubit Isospin Precession, Phys. Rev. B 89, 195405 (2014).
[85] M. Hell, M. R. Wegewijs, and D. P. DiVincenzo, Qubit Quantum-Dot Sensors: Noise Cancellation by Coherent Backaction, Initial Slips, and Elliptical Precession, Phys. Rev. B 93, 045418 (2016).

[86] H.-P. Breuer, E.-M. Laine, and J. Piilo, Measure for the Degree of Non-Markovian Behavior of Quantum Processes in Open Systems, Phys. Rev. Lett. 103, 210401 (2009).

[87] F. Buscemi and N. Datta, Equivalence between Divisibility and Monotonic Decrease of Information in Classical and Quantum Stochastic Processes, Phys. Rev. A 93, 012101 (2016).

[88] H.-P. Breuer, E.-M. Laine, J. Piilo, and B. Vacchini, Colloquium: Non-Markovian Dynamics in Open Quantum Systems, Rev. Mod. Phys. 88, 021002 (2016).

[89] A. Smirne, L. Mazzola, M. Paternostro, and B. Vacchini, Interaction-Induced Correlations and NonMarkovianity of Quantum Dynamics, Phys. Rev. A 87, 052129 (2013).

[90] F. Benatti, D. Chruściński, and S. Filippov, Tensor Power of Dynamical Maps and Positive versus Completely Positive Divisibility, Phys. Rev. A 95, 012112 (2017).

[91] H.-P. Breuer, G. Amato, and B. Vacchini, Mixing-Induced Quantum Non-Markovianity and Information Flow, New J. Phys. 20, 043007 (2018).

[92] D. Chruściński, C. Macchiavello, and S. Maniscalco, Detecting Non-Markovianity of Quantum Evolution via Spectra of Dynamical Maps, Phys. Rev. Lett. 118, 080404 (2017).

[93] J. Schulenborg, R. B. Saptsov, F. Haupt, J. Splettstoesser, and M. R. Wegewijs, Fermion-Parity Duality and Energy Relaxation in Interacting Open Systems, Phys. Rev. B 93, 081411(R) (2016).

[94] V. Bruch, K. Nestmann, M. R. Wegewijs, and J. Schulenborg, Fermionic Duality: General Symmetry of Open Systems with Strong Dissipation and Memory, arXiv:2104.11202.

[95] U. Geigenmüller, U. M. Titulaer, and B. U. Felderhof, Systematic Elimination of Fast Variables in Linear Systems, Physica (Amsterdam) 119A, 41 (1983).

[96] F. Haake and M. Lewenstein, Adiabatic Drag and Initial Slip in Random Processes, Phys. Rev. A 28, 3606 (1983).

[97] F. Haake and R. Reibold, Strong Damping and LowTemperature Anomalies for the Harmonic Oscillator, Phys. Rev. A 32, 2462 (1985).

[98] P. Gaspard and M. Nagaoka, Slippage of Initial Conditions for the Redfield Master Equation, J. Chem. Phys. 111, 5668 (1999).

[99] T. Yu, L. Diósi, N. Gisin, and W. T. Strunz, Post-Markov Master Equation for the Dynamics of Open Quantum Systems, Phys. Lett. A 265, 331 (2000).

[100] B. M. Garraway, Decay of an Atom Coupled Strongly to a Reservoir, Phys. Rev. A 55, 4636 (1997).

[101] L. Mazzola, S. Maniscalco, J. Piilo, K.-A. Suominen, and B. M. Garraway, Pseudomodes as an Effective Description of Memory: Non-Markovian Dynamics of Two-State Systems in Structured Reservoirs, Phys. Rev. A 80, 012104 (2009). 
[102] V. Reimer, M. R. Wegewijs, K. Nestmann, and M. Pletyukhov, Five Approaches to Exact Open-System Dynamics: Complete Positivity, Divisibility, and Time-Dependent Observables, J. Chem. Phys. 151, 044101 (2019).

[103] D. Chruściński and A. Kossakowski, Non-Markovian Quantum Dynamics: Local versus Nonlocal, Phys. Rev. Lett. 104, 070406 (2010).

[104] D. Chruściński, Á. Rivas, and E. Størmer, Divisibility and Information Flow Notions of Quantum Markovianity for Noninvertible Dynamical Maps, Phys. Rev. Lett. 121, 080407 (2018).

[105] S. Chakraborty and D. Chruściński, Information Flow versus Divisibility for Qubit Evolution, Phys. Rev. A 99, 042105 (2019).

[106] U. Chakraborty and D. Chruściński, Construction of Propagators for Divisible Dynamical Maps, arXiv: 2004.09264.

[107] The limit $t-t_{0} \rightarrow \infty$ converges for the examples in Sec. V, but in general requires care and is beyond the present scope.

[108] Note that $\left.\mid g_{i}\right)=\hat{g}_{i}$ corresponds to an operator $\hat{g}_{i}$ and that $\left(\bar{g}_{i} \mid \bullet=\operatorname{Tr}\left[\hat{\bar{g}}_{i}^{\dagger} \cdot\right]\right.$ corresponds to a different operator $\hat{\bar{g}}_{i}-$ indicated by the bar-such that $\left(\bar{g}_{i} \mid g_{j}\right)=\operatorname{Tr}\left[\hat{\bar{g}}_{i}^{\dagger} \hat{g}_{j}\right]=\delta_{i j}$.

[109] S. Andergassen, M. Pletyukhov, D. Schuricht, H. Schoeller, and L. Borda, A Renormalization-Group Analysis of the Interacting Resonant Level Model at Finite Bias: Generic Analytic Study of Static Properties and Quench Dynamics, Phys. Rev. B 83, 205103 (2011).

[110] Here we are assuming for simplicity that all poles of $\hat{\Pi}(\omega)$ are first-order poles. For a detailed discussion, see Ref. [94].

[111] A. J. van Wonderen and L. G. Suttorp, Kraus Map for NonMarkovian Quantum Dynamics Driven by a Thermal Reservoir, Eur. Phys. Lett. 102, 60001 (2013).

[112] A. J. van Wonderen and L. G. Suttorp, Continued-Fraction Representation of the Kraus Map for Non-Markovian Reservoir Damping, J. Phys. A 51, 175304 (2018).

[113] A. J. van Wonderen and L. G. Suttorp, Exact Density Matrix of a Discrete Quantum System Immersed in a Thermal Reservoir, arXiv:1808.04198.

[114] Note that $\Pi(t)$ is CP divisible $[39,41,52]$ if $\mathcal{G}(t)$ has nonnegative GKSL coefficients for all times $t$. We allow for negative coefficients at finite times. Models where the evolution has negative asymptotic GKSL coefficients have also been studied recently $[19,42,115]$.

[115] K. Siudzińska and D. Chruściński, Quantum Evolution with a Large Number of Negative Decoherence Rates, arXiv:2006.02793.

[116] F. Cavaliere, M. Governale, and J. König, Nonadiabatic Pumping through Interacting Quantum Dots, Phys. Rev. Lett. 103, 136801 (2009).

[117] M. A. Nielsen and I. L. Chuang, Quantum Computation and Quantum Information (Cambridge University Press, Cambridge, England, 2010).

[118] The generator can be written in time-dependent GKSL form $-i \mathcal{G}(t)=-i[H, \bullet]+j J \bullet J^{\dagger}$, with $H=\varepsilon|1\rangle\langle 1|$ and jump operator $J=|0\rangle\langle 1|$. For $\gamma<2 \Gamma$, the jump rate $j=$ $-2 \operatorname{Re}[\dot{\pi}(t) / \pi(t)]$ can be negative by Eq. (46) which is equivalent to $\mathrm{CP}$ divisibility $[39,41]$. For $\gamma \geq 2 \Gamma$, we have $j>0$ by $\dot{\pi}(t) / \pi(t)=-i \varepsilon-\frac{1}{2} \gamma+\frac{1}{2} \gamma^{\prime} \tanh \left(\frac{1}{2} \gamma^{\prime} t+\tanh ^{-1} \frac{\gamma}{\gamma^{\prime}}\right)$.

[119] (i) Select any four poles $\omega_{s_{1}}, \ldots, \omega_{s_{4}}$ from Table I that have linearly independent right eigenvectors. These are eigenvectors from different superoperators $\hat{\mathcal{K}}\left(\omega_{i}^{\prime}\right)$ and thus need not be linearly independent. (ii) From this basis, $\left.\left.\mid k_{s_{1}}\right), \ldots, \mid k_{s_{4}}\right)$ construct a corresponding dual basis $\left(\bar{k}_{s_{1}} \mid, \ldots,\left(\bar{k}_{s_{4}} \mid\right.\right.$. (iii) Construct a fixed point as $\mathcal{G}_{s_{1} \ldots s_{4}}=$ $\left.\sum_{i=1}^{4} \omega_{s_{i}} \mid k_{s_{i}}\right)\left(\bar{k}_{s_{i}} \mid\right.$.

[120] T. Yu and J. H. Eberly, Finite-Time Disentanglement via Spontaneous Emission, Phys. Rev. Lett. 93, 140404 (2004).

[121] M. P. Almeida, F. de Melo, M. Hor-Meyll, A. Salles, S. P. Walborn, P. H. S. Ribeiro, and L. Davidovich, Environment-Induced Sudden Death of Entanglement, Science 316, 579 (2007).

[122] J. Laurat, K. S. Choi, H. Deng, C. W. Chou, and H. J. Kimble, Heralded Entanglement between Atomic Ensembles: Preparation, Decoherence, and Scaling, Phys. Rev. Lett. 99, 180504 (2007).

[123] T. Yu and J. H. Eberly, Sudden Death of Entanglement, Science 323, 598 (2009).

[124] H.-P. Breuer, B. Kappler, and F. Petruccione, Stochastic Wave-Function Method for Non-Markovian Quantum Master Equations, Phys. Rev. A 59, 1633 (1999).

[125] This relation should not be misunderstood as saying that " $\mathcal{G}(\infty)$ only samples $\mathcal{K}(\omega)$ at $\omega=0$ " and that there is something trivial about the sampling: keeping only $\omega=0$ in Eq. (21) would give $\mathcal{G}(\infty)=0$. Instead, this relation is a nontrivial statement about the memory kernel of this model: sampling $\hat{\mathcal{K}}(\omega)$ according to Eq. (21) at eigenvalue poles-including ones at nonzero frequencies and ignoring eigenvector poles or branch cuts-exactly reproduces the zero-frequency kernel $\hat{\mathcal{K}}(0)$.

[126] A. Oppenheim, A. S. Willsky, and I. Young, Signals and Systems (Prentice-Hall, New York, 1983).

[127] T. Pluecker, M. R. Wegewijs, and J. Splettstoesser, Meter Calibration and the Geometric Pumping Process in Open Quantum Systems, arXiv:1711.10431.

[128] C.Z. Ning and H. Haken, Geometrical Phase and Amplitude Accumulations in Dissipative Systems with Cyclic Attractors, Phys. Rev. Lett. 68, 2109 (1992).

[129] A. S. Landsberg, Geometrical Phases and Symmetries in Dissipative Systems, Phys. Rev. Lett. 69, 865 (1992).

[130] A. S. Landsberg, Spatial Symmetries and Geometrical Phases in Classical Dissipative Systems, Mod. Phys. Lett. B 07, 71 (1993).

[131] T. Pluecker, M. R. Wegewijs, and J. Splettstoesser, Gauge Freedom in Observables and Landsberg's Nonadiabatic Geometric Phase: Pumping Spectroscopy of Interacting Open Quantum Systems, Phys. Rev. B 95, 155431 (2017).

[132] N. A. Sinitsyn, Reversible Stochastic Pump Currents in Interacting Nanoscale Conductors, Phys. Rev. B 76, 153314 (2007).

[133] Y. V. Nazarov and M. Kindermann, Full Counting Statistics of a General Quantum Mechanical Variable, Eur. Phys. J. B 35, 413 (2003). 
[134] G. Schaller, G. Kießlich, and T. Brandes, Transport Statistics of Interacting Double Dot Systems: Coherent and NonMarkovian Effects, Phys. Rev. B 80, 245107 (2009).

[135] G. Guarnieri, C. Uchiyama, and B. Vacchini, Energy Backflow and Non-Markovian Dynamics, Phys. Rev. A 93, 012118 (2016).

[136] Y. Utsumi, Full Counting Statistics of Information Content and Particle Number, Phys. Rev. B 96, 085304 (2017).

[137] S. Nakajima and Y. Tokura, Excess Entropy Production in Quantum System: Quantum Master Equation Approach, J. Stat. Phys. 169, 902 (2017).

[138] A. Müller-Hermes and D. Reeb, Monotonicity of the Quantum Relative Entropy under Positive Maps, Ann. Inst. Henri Poincaré 18, 1777 (2017).
[139] M. S. Ferguson, O. Zilberberg, and G. Blatter, Open Quantum Systems beyond Fermi's Golden Rule: Diagrammatic Expansion of the Steady-State Time-Convolutionless Master Equation, arXiv:2010.09838.

[140] K. G. Wilson, The Renormalization Group: Critical Phenomena and the Kondo Problem, Rev. Mod. Phys. 47, 773 (1975).

[141] One verifies Eq. (B6a) first for $n=2$, giving $F_{p_{1} p_{2}}^{2}=$ $F_{\left(p_{1}-1\right) p_{2}}^{2}+F_{p_{1}\left(p_{2}-1\right)}^{2}$, and uses this relation for $n>2$ to simplify the sum of the last two terms, $F_{p_{1} \ldots\left(p_{n-1}-1\right) p_{n}}^{n}+$ $F_{p_{1} \ldots p_{n-1}\left(p_{n}-1\right)}^{n}$. Then one adds $F_{p_{1} \ldots\left(p_{n-2}-2\right) p_{n-1} p_{n}}^{n}$, and so forth. Equation (B6b) follows as a special case of Eq. (B6a) since $F_{p_{1} . p_{n-1}(-1)}^{n}=F_{p_{1} \ldots p_{n-1}}^{n-1}$. 